\title{
MET Oncogene in Non-Small Cell Lung Cancer: Mechanism of MET Dysregulation and Agents Targeting the HGF/c-Met Axis
}

This article was published in the following Dove Press journal: OncoTargets and Therapy

\section{Hongge Liang (D) \\ Mengzhao Wang}

Peking Union Medical College Hospital, Chinese Academy of Medical Sciences \& Peking Union Medical College, Beijing 100730, People's Republic of China
Correspondence: Mengzhao Wang Peking Union Medical College Hospital, Chinese Academy of Medical Sciences \& Peking Union Medical College, No. I Shuaifuyuan, Dongcheng District, Beijing 100730, People's Republic of China Tel +86 I0-69l55039

Email mengzhaowang@sina.com

\begin{abstract}
Non-small cell lung cancer (NSCLC) is the leading cause of cancer-related death worldwide and has a poor prognosis. Current treatments for advanced NSCLC included traditional chemotherapy, radiotherapy, targeted therapy, and immunotherapy. The efficacy of targeted therapy relies on oncogene addiction. Mesenchymal-epithelial transition factor (MET) gene can encode unconventional receptor tyrosine kinases with pleiotropic functions, when signals are abnormally activated, it can initiate and maintain tumor transformation, promote cell proliferation, survival, tumor invasion and angiogenesis. Thus, it is a promising therapeutic target. Previous studies have shown that elevated levels of HGF and/or overexpression of c-Met are associated with poor prognosis in lung cancer. In preclinical and clinical trials, c-MET inhibitors have shown some antitumor activity in NSCLC. Although the efficacy results of MET inhibitors in Phase III clinical trials are disappointing, given the molecular heterogeneity of NSCLC, only subgroups of patients with MET gene alterations may benefit from c-MET inhibitors. The challenge for the future is to screen out the potential beneficiaries. To solve this problem, there is need for large data analysis for the detection methods and treatment effects, to establish standards that meet the MET activation status, and determine reliable thresholds to achieve effective patient stratification and clinical decision making. This article summarized the structure of the hepatocyte growth factor (HGF)/c-Met axis, the different mechanisms of MET addiction, as well as MET amplification as acquired resistance mechanism to epidermal growth factor receptor-tyrosine kinase inhibitors, the latest advances of MET inhibitors, and immuotherapy in the treatment of NSCLC with MET alterations.
\end{abstract}

Keywords: c-mesenchymal-epithelial transition, receptor tyrosine kinases, non-small cell lung cancer, treatment, oncogene addiction

\section{Introduction}

Non-small cell lung cancer (NSCLC) is the leading cause of cancer-related death worldwide. ${ }^{1}$ Most patients with NSCLC are diagnosed at an advanced stage, and traditional chemotherapy and radiotherapy have shown limited efficacy. ${ }^{2}$ Although immunotherapy has changed the current state of treatment for NSCLC, many patients do not respond to programmed cell death protein-1 (PD-1)/ programmed death-ligand 1 (PD-L1) inhibitors, especially in patients harboring driven mutations. Small molecule tyrosine kinase inhibitors (TKIs) are now approved for treatment in patients with NSCLC harboring epidermal growth factor receptor (EGFR) mutations, BRAF V600E mutations, anaplastic lymphoma kinase (ALK) rearrangements, and ROS1 
rearrangements; however, all patients will inevitably develop progression of disease. ${ }^{3}$ Therefore, it is necessary to find new therapeutic targets that drive the pathogenesis of NSCLC and develop more effective targeted drugs.

C-mesenchymal-epithelial transition factor (c-MET), the hepatocyte growth factor (HGF) receptor, is an oncogene encoding tyrosine kinase receptor. It mainly exists in epithelial cells, and plays an important role in embryogenesis, tumor growth, and metastasis. ${ }^{4}$ Once tyrosine kinase receptors are activated by their ligands, mitosis is triggered. This can regulate a variety of cellular functions. ${ }^{5,6}$ The dysregulation of MET/HGF axis pathway is involved in the proliferation, survival, invasion, and metastasis of tumor cells. ${ }^{7,8}$ This can be found in NSCLC and other solid tumors such as breast cancer, cervical cancer, stomach cancer, and colon cancer. ${ }^{9,10}$ C-MET alterations in NSCLC include point mutations, amplification, fusion, and protein overexpression, which are associated with poor prognosis. ${ }^{11-15}$ Previous preclinical and clinical studies suggested that MET activation is both a primary oncogenic driver mutation and a secondary driver of acquired resistance to targeted therapy in other genomic subpopulations. ${ }^{16}$ Therefore, agents targeting c-MET are a promising treatment strategy for NSCLC. At present, a number of pre-clinical and clinical studies have been conducted on many drugs targeting MET (small molecule TKI, MET antibody, and HGF antibody). 5,17,18 This article reviewed the mechanisms of MET gene addiction, and the clinical application of MET inhibitors in NSCLC.

\section{Dysregulated c-MET Signaling in NSCLC}

The c-MET gene is located on chromosome 7 q21-31 belonging to the HGF receptor family, which encodes a protein tyrosine kinase, and regulates important cellular processes including cell differentiation, proliferation, cell cycle, movement, and apoptosis. HGF is a paracrine-signaling molecule that is produced and secreted by mesenchymal cells, which is the only ligand for c-MET. ${ }^{19}$ The extracellular portion of c-MET consists of the immunoglobulin (Ig)-like, plexins, transcription factors (IPT) domain, the plexin-semaphorinintegrin (PSI) domain, and the Sema domain (homologous to semaphorin) responsible for binding to HGF. The intracellular portion of c-MET consists of the juxtamembrane (JM) domain, the Catalytic domain, and the Docking site responsible for signal transduction (Figure 1). HGF/c-MET binding leads to receptor dimerization, tyrosine residues autophosphorylate, substrate docking, and activation of downstream signaling pathways such as PI3K/AKT, RAS/ERK/MAPK, Wnt/B-catenin, SRC, and STAT320-29; ${ }^{20-29}$ thereby, inducing

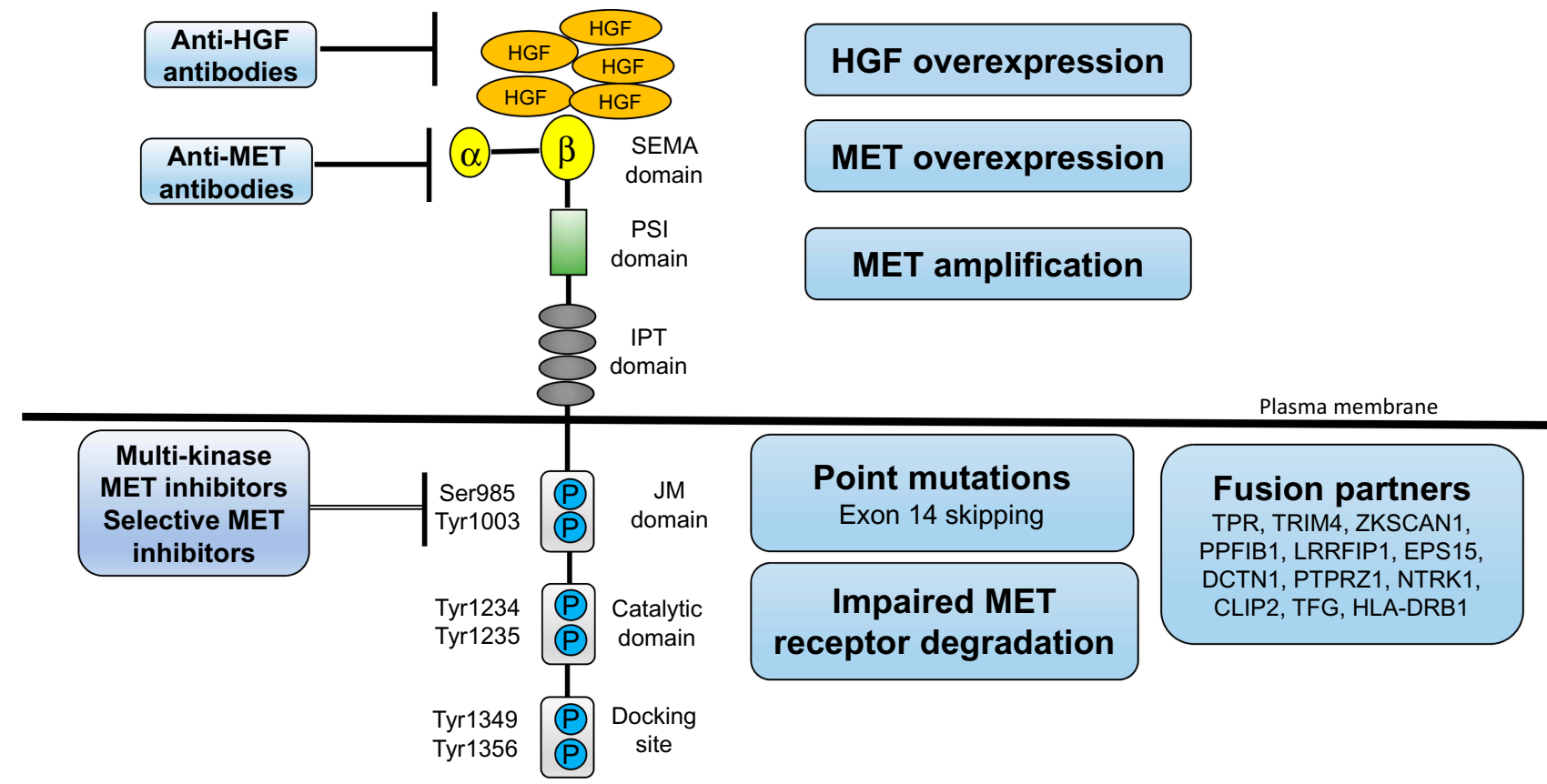

Figure I Major Mechanism of MET/HGF axis dysregulation. The extracellular portion of c-MET consists of a four immunoglobulin (lg)-like modules, a cysteine-rich, METrelated sequence domain, and a Sema domain (homologous to semaphorin) responsible for binding to HGF. The intracellular portion of c-MET consists of the paramembrane domain, the Catalytic domain, and the Docking site responsible for signal transduction. Various mechanisms of MET/HGF axis addiction in NSCLC, including MET/HGF overexpression, and MET gene alterations (including point mutations, amplification, and fusion). A number of drugs targeting MET (small molecule TKIs, MET antibody, and HGF antibody) have been studied. MET, c-mesenchymal-epithelial transition factor; HGF, hepatocyte growth factor; NSCLC, non-small cell lung cancer. 
excessive cell proliferation, and is closely related to the occurrence and development of tumors. It is reported that the deregulation of the MET signaling in NSCLC can induce tumor invasion and metastasis, ${ }^{30}$ and can interact with other signaling pathways such as EGFR. ${ }^{31,32}$

\section{MET Exon 14 Skipping Mutations}

METex14 alterations (point mutations, deletions, insertions, and complex mutations) lead to decreased degradation of MET receptor, resulting in the activation of MET signaling and the tumorigenesis. ${ }^{33-36}$ The evaluation methods for METex14 skipping mutations include differential MET exon expression, ${ }^{37,38}$ quantitative reverse transcriptionpolymerase chain reaction (qRT-PCR) ${ }^{39}$ or direct RNA sequencing. ${ }^{37,40}$ In addition, DNA and RNA sequencing can be used to detect Y1003, a "functional mimetic" of the METex14 skipping mutation, accounting for approximately $2 \%$ of all METex14 alterations. ${ }^{41}$

In 2015, Frampton et al performed a large-scale molecular profiling of METex14 alterations in 38,028 tumor samples. Of the 221 tumor samples containing METex14 alterations, 193 were found to be lung cancer, of which 131 were lung adenocarcinoma. $^{42}$ METex14 skipping mutations have been reported in $2-4 \%$ of NSCLC. ${ }^{37,43-45}$ The mutation rate was closely related to the histological subtype of NSCLC, most commonly in sarcomatoid carcinoma $(4.9 \% \sim 31 \%)$, followed by adenosquamous carcinoma $(5 \%)$, adenocarcinoma $(3 \%)$ and squamous cell carcinoma $(2 \%){ }^{39,41,46-48}$ In addition, METex14 mutations are likely to be mutually exclusive of other genetic alterations in NSCLC and are more likely to occur in elderly, non-smoker patients. ${ }^{39,42}$ In a study of 933 patients with non-squamous NSCLC, no mutations in the KRAS, EGFR, erb-b2 receptor tyrosine kinase 2 gene (ERBB2), ALK, ROS1, or RET were found in 30 patients with METex14 alterations. ${ }^{39}$ In 687 Asian patients with NSCLC, METex14 skipping mutations are poor prognostic factors of overall survival. ${ }^{49}$ In NSCLC patients, METex14 skipping mutations are associated with the efficacy of MET protein TKIs, especially in patients with advanced lung sarcomatoid carcinomas. ${ }^{38,42,48,50-54}$

\section{MET Amplification}

MET amplification is the carcinogenic driver. Fluorescence in situ hybridization (FISH) can distinguish between the two based on the ratio of MET/CEP7 (centromeric chromosome 7). In polyploid, each copy of MET is associated with centromeres, and the MET/CEP7 ratio remains constant as the number of copies increases. In MET amplification, the
MET/CEP7 ratio increased due to the increased copy number of the MET gene. ${ }^{55}$ Next generation sequencing can also be used to evaluate MET amplification and provide other potentially clinically relevant genomic alteration information. ${ }^{56}$

The MET amplification was reported in $2-5 \%$ of NSCLC. ${ }^{57,58}$ The high MET gene amplification (MET/CEP7 $\geq 5$ ) is very rare, with an incidence of only $0.34 \%$, and no other oncogenic driver genes were found in these patients compared to patients with low MET gene amplification (MET/CEP7 ratio $<5$ ), which also suggests that high MET gene amplification may act as a carcinogenic driver. ${ }^{59}$ In addition, the incidence of MET amplification was higher in NSCLC patients treated with erlotinib/gefitinib, ranging from 5\% to $22 \%$. $31,32,60$ MET FISH-positive patients with advanced NSCLC have a poor overall survival. ${ }^{13,14,61}$ There is a correlation between high amplification of MET gene and high response rate of crizotinib. $^{62}$ In a Phase I clinical trial (NCT00585195), a patient with advanced lung adenocarcinoma harboring high levels of MET amplification by FISH (MET/CEP7>5), and received crizotinib treatment, obtained a persistent partial response (PR). ${ }^{63}$ Preliminary results showed that $1(16.7 \%)$ of 6 patients with moderate MET amplification (MET/CEP7 ratio $>2.2$ to $<5)$ obtained $\mathrm{PR}$; and $3(50 \%)$ of 6 patients with high-level MET amplification (MET/CEP7 ratio >5) obtained PR. No PR was observed in patients with low MET amplification levels (MET/CEP7 ratio 1.8 to 2.2). ${ }^{64}$ Notably, Zhang et al reported a rapid response to crizotinib in a patient with lung adenocarcinoma, which showed an increase in MET copy number but a low MET/CEP7 ratio. ${ }^{65}$ Therefore, larger clinical trials are needed to assess the predictive value of MET gene amplification for targeted therapies.

\section{Impaired MET Receptor Degradation}

Impaired MET receptor degradation seems to be a mechanism for ligand-independent aberrant MET signaling. ${ }^{66}$ The $\mathrm{HGF} / \mathrm{SF}$ binding to MET induces dimerization of the c-MET receptor and activation of downstream signaling pathways. Tyrosine 1003 (Y1003), contained in c-MET juxtamembrane domain encoded by METex14, is a direct binding site for c-Cbl, an E3 ubiquitin ligase. After c-Cbl binds to Y1003, the MET receptor is internalized into endosomes and ubiquitinated, and degraded by the lysosomal pathway. ${ }^{35}$ However, in NSCLC, nearly all of the METex14 skipping can delete Y1003, c-Cbl binding site, in the juxtamembrane domain. ${ }^{42}$ This leads to MET ubiquitination abrogation, increased MET protein stability, and impaired MET degradation. This induces ligand-independent MET activation. ${ }^{35,42,66,67}$ Besides METex14 skipping, mutant cells of Casitas B-lineage 
lymphoma (CBL), an E3 ubiquitin ligase, show decreased MET ubiquitination, increased MET expression, and higher sensitivity to MET inhibitor SU11274 than CBL wild-type cells, suggesting that CBL status maybe a potential biomarker for MET-targeted therapeutics in NSCLC. ${ }^{68}$

\section{MET Fusion}

The MET fusion gene consists of METs that are lacking in juxtamembrane regulatory sequence and different $\mathrm{N}$-terminal partners. The genes that are currently found to be fused with MET include TPR, TRIM4, ZKSCAN1, PPFIB1, LRRFIP1, EPS15, DCTN1, PTPRZ1, NTRK1, CLIP2, TFG, HLA-DRB1, etc. These chimeric proteins are constitutively phosphorylated in a xenograft animal model and induce tumorigenesis. ${ }^{69} \mathrm{~A}$ case report described a patient with lung cancer with HLA-DRB1-MET fusion who obtained PR for 8 months after receiving crizotinib. ${ }^{70}$ As with the fusions described above, this chimeric MET contains a kinase domain without a juxtamembrane regulatory sequence. Another kinase fusion, KIF5B-MET, was found in a patient with lung adenocarcinoma. This fusiondriven MET activation is probably due to constitutive dimerization and is likely an operational target which can be inhibited by agents. It is similar to other fusions in lung cancer, such as ALK, ROS1, and RET. ${ }^{71}$

\section{Overexpression of MET/p-MET}

In addition to gene amplification, mutation or fusion, the MET pathway can also activate protein overexpression by upregulating the secretion signal of MET or HGF, inducing tumor transformation. ${ }^{72,73}$ The MET overexpression rate in unselected NSCLC ranges from $15 \%$ to $70 \%$, which depends on the antibody assay and positive threshold. ${ }^{10,74-76}$ In addition to the total level of protein, ligand-activated MET that induces phosphorylation of the juxtamembrane domain can also be detected by phosphorylation-MET (p-MET). Specific expression of p-MET has been observed in approximately two-thirds of lung cancer samples. ${ }^{10,36}$ Previous study has reported the expression and prognosis value of MET, p-MET, and HGF in lung cancer patients $(n=129) .{ }^{77}$ The high expression of two specific forms of p-MET, cytoplasmic expression of Y1003 $(\mathrm{P}=0.016 ; \mathrm{HR}=1.86 ; 95 \% \mathrm{CI}: 1.12-3.07)$, and nuclear expression of $\mathrm{Y} 1365(\mathrm{P}=0.034 ; \mathrm{HR}=1.70 ; 95 \%$ CI: 1.04-2.78) were negative prognostic factors for overall survival. Thus, the particular type of p-MET may be biomarkers for selecting patients who are likely to benefit from MET inhibitors.

Although the overexpression of MET protein is associated with poor prognosis in lung cancer, it was less effective as a predictive biomarker for targeted therapeutic efficacy. ${ }^{78}$ Previous studies have found that MET overexpression has higher sensitivity and negative predictive value $;{ }^{49,79}$ however, due to the significant intratumoral heterogeneity, immunohistochemical evaluation of MET overexpression remains challenging, limiting its use as a biomarker in the clinic. ${ }^{80,81}$ This difficulty in screening patients for MET-targeted therapy may also partly explain the failure of MET inhibitors in recent phase III clinical trials of NSCLC. ${ }^{82,83}$

\section{Role of MET in Induction of EGFR-TKIs Resistance}

MET amplification is a potential resistance pattern of EGFR-TKIs in NSCLC, accounting for $50-60 \%$ of the first- and second-generation EGFR-TKIs acquired resistance, ${ }^{31,32,84}$ accounting for $15-19 \%$ of the thirdgeneration EGFR-TKIs acquired resistance. ${ }^{85,86}$ In 2018, ESMO reported two important studies on the mechanisms of acquired resistance to osimertinib. ${ }^{85,86}$ FLAURA and AURA2 study provided patients treated with first-line and second-line osimertinib, respectively. Secondgeneration sequencing was performed on patient plasma samples at disease progression and/or treatment discontinuation. The results showed that MET amplification was the most common acquired resistance mechanism to firstline (15\% MET amplification, $\mathrm{n}=91)$ and second-line $(19 \%$ MET amplification, $n=73$ ) treatment. In addition, increased circulating levels of HGF in tumor and stromal cells can lead to dysregulation of the MET pathway, ${ }^{87,88}$ which can also confer EGFR-TKIs resistance. ${ }^{87,89}$ In vitro and in vivo preclinical studies have shown that MET-targeted agents can reverse the resistance to EGFR inhibitors and restore sensitivity in this particular genetic context. ${ }^{32,90,91}$

In fact, synergistic effects of epidermal growth factor (EGF) and HGF on cell proliferation have been demonstrated in preclinical studies of NSCLC. When cells are stimulated by HGF and EGF, respectively, an increase in cell wrinkles can be observed, to form on the mobile cell surface. When these growth factors are combined, an additive effect can be observed, ${ }^{92}$ indicating that the combination of MET inhibitors and EGFR-TKIs may have synergistic anti-tumor effects. This provide a theoretical basis for the clinical application of combination targeted therapy in the treatment of patients with NSCLC, especially of patients with EGFR-TKIs resistance.

\section{Therapeutics to Inhibit the HGF/c-Met Axis}

Only patients with MET gene alterations may have an objective response (tumor regression) after receiving MET-targeted therapy, whereas targeting the HGF/MET axis in MET wild- 
type patients has little effect on cancer cell growth or on the efficacy of chemotherapy agents. ${ }^{93}$ In many cases, MET inhibitors have been reported to be effective in patients with NSCLC, who have high levels of MET amplification ${ }^{63,64}$ or METex14 skipping mutations. ${ }^{38,42,51,54,94-96}$ There are a variety of MET inhibitors, including small molecule TKIs and monoclonal antibodies (mAbs) against MET or its ligand HGF, for clinical studies of NSCLC. The results of clinical studies of c-MET inhibitors for NSCLC are shown in Table 1. Table 2 summarizes ongoing clinical studies on c-MET inhibitors for NSCLC.

\section{Multi-Kinase MET Inhibitors Crizotinib (PF-0234I066)}

Crizotinib is an oral multi-target TKI that competes with c-Met tyrosine kinase domain for binding the adenosine triphosphate (ATP) site, ${ }^{30}$ preventing receptor activation and downstream signaling transmission, as well as competing for ATP binding sites with other kinases, including vascular endothelial growth factor receptor (VEGFR), ROS1, and EML4-ALK, etc. ${ }^{64,97,98}$ Crizotinib has been approved by the FDA for patients with advanced NSCLC harboring ALK or ROS1 fusion. ${ }^{99,100}$

In PROFILE-1001 study, of 18 patients with NSCLC harbored METex14 skipping mutations who received crizotinib, 8 patients obtained an objective response (44\%; 95\% CI: $22-69 \%) ;^{50} 13$ patients with c-MET amplification were divided into low (1.8-2.2), medium $(>2.2-<5)$, and high $(\geq 5)$ FISH ratio grades. The results showed that 4 patients obtained an objective response $(33 \%$; 95\% CI: $10-65 \%)$. One of the four was mid-level while three were of high-level amplification group ${ }^{64}$ A multicenter retrospective analysis showed that for patients with METex14 NSCLC, treatment with a MET inhibitor (including crizotinib) is associated with an improvement in overall survival. The median overall survival (mOS) of 34 metastatic patients, who had never received MET inhibitor therapy, was 8.1 months, while mOS of 27 metastatic patients who had received at least one MET inhibitor (including crizotinib, glesatinib, capmatinib, and ABBV399), was 24.6 months. Treatment with MET inhibitors significantly prolonged these patients' $\operatorname{mOS}(\mathrm{HR}=0.11$; 95\% CI: $0.01-0.92 ; \mathrm{p}=0.04)$. The median progressionfree survival (PFS) of 22 patients receiving crizotinib was 7.36 months. ${ }^{101}$ In addition, previous studies have also evaluated the efficacy and safety of crizotinib in combination with other targeted therapies in patients with NSCLC, including erlotinib ${ }^{102}$ and dacomitinib. ${ }^{103}$ However, combination therapy leads to many adverse events (43\% of patients had grade 3 or 4 treatment-related adverse events [AEs]) and the efficacy was poor. With these poor results, there are no plans for further research.

Crizotinib has clinical activity in patients with NSCLC with METex14 and MET amplification (FISH ratio grades $>5$ ), indicating that METex14 evaluated by NGS and MET amplification evaluated by FISH appears to be biomarkers for crizotinib treatment in patients with NSCLC. This needs further confirmation. Besides, due to the adverse effects and limited efficacy, combination of crizotinib with other targeted agents is not recommended.

\section{Cabozantinib (XL-I84, BMS-90735I)}

Cabozantinib is another multi-kinase inhibitor targeting c-Met, VEGFR1, VEGFR2, VEGFR3, RET, TIE2, FLT-3 and KIT, which blocks a variety of precancerous signaling pathways. $^{104}$ It has significant oral bioavailability and blood-brain barrier penetration.

A phase I clinical trial analyzed the efficacy and safety of cabozantinib in patients with various solid tumors including NSCLC. ${ }^{105}$ The results showed that in NSCLC patients $(n=60)$, the objective response rate was $10 \%$, and the median PFS was 4.0 months. Another study evaluated the efficacy of cabozantinib in combination with erlotinib in patients with EGFR-mutant NSCLC who were resistant to EGFR-TKIs. ${ }^{106}$ The results showed that the objective response rate was $10.8 \%(95 \% \mathrm{CI}$ 0.3-21.3\%), median PFS was 3.6 months (95\% CI: 2.0-5.6), and the mOS was 13.1 months (95\% CI 7.1-NA). Recently, a randomized Phase II trial evaluated the efficacy of erlotinib monotherapy, cabozantinib monotherapy, and erlotinib, in combination with cabozantinib in patients with EGFR wild-type NSCLC. ${ }^{107}$ There were 111 patients $(38,38,35)$ included in the preliminary analysis. The results showed a significant improvement in the median PFS between the cabozantinib group (4.3 vs 1.8; HR: $0.39, \mathrm{p}=0.0003$ ) and the combination treatment group (4.7 vs 1.8; HR: $0.37, \mathrm{p}=0.0003$ ) compared with the erlotinib group, with tolerable safety.

In clinical trials related to cabozantinib, MET alterations have not been evaluated, but mainly focused on the EGFR status. The theoretical basis is that MET alterations, especially MET amplification may be a potential acquired resistance mechanism to EGFR-TKIs. All these results indicate that cabozantinib alone or in combination with EGFR-TKIs has a potential research prospect for NSCLC patients with EGFR wild-type or resistance to EGFRTKIs. 
Table I Results of Clinical Trials with Targeted MET Inhibitors in NSCLC

\begin{tabular}{|c|c|c|c|c|c|c|c|c|}
\hline $\begin{array}{l}\text { Agents \& } \\
\text { Mechanism }\end{array}$ & Study & phase & Patients & c-MET Alteration & Treatment & $\begin{array}{l}\text { No. } \\
\text { of } \\
\text { Pts }\end{array}$ & Efficacy Outcome & Status \\
\hline \multirow[t]{2}{*}{$\begin{array}{l}\text { Crizotinib } \\
\text { (PF0234I066) } \\
\text { Multi-TKls }\end{array}$} & $\begin{array}{l}\text { NCT00585195 } \\
\text { (PROFILE- } \\
\text { I00I) }\end{array}$ & 1 & NSCLC & $\begin{array}{l}\text { MET exon I4- } \\
\text { altered }\end{array}$ & Crizotinib & 18 & $\begin{array}{l}\text { ORR: } 44 \%(22-69 \%) \\
\text { Crizotinib has antitumor } \\
\text { activity in patients with MET } \\
\text { exon 14-altered NSCLC. }\end{array}$ & $\begin{array}{l}\text { Active, not } \\
\text { recruiting }\end{array}$ \\
\hline & NCT00585195 & 1 & NSCLC & $\begin{array}{l}\text { c-Met-amplification } \\
\text { (FISH) }\end{array}$ & Crizotinib & 13 & $\begin{array}{l}\text { ORR: } \\
\text { MET/CEP } 7 \geq 1.8: 33 \%(n=13) \\
2.2<\text { MET/CEP7<5: } 20 \%(n=6) \\
\text { MET/CEP } 7 \geq 5: 50 \%(n=6)\end{array}$ & $\begin{array}{l}\text { Active, not } \\
\text { recruiting }\end{array}$ \\
\hline \multirow[t]{3}{*}{$\begin{array}{l}\text { Cabozantinib } \\
(\mathrm{XLI84)} \\
\text { Multi-TKIs }\end{array}$} & NCT0I8664I0 & 2 & $\begin{array}{l}\text { Pretreated } \\
\text { EGFR-mutation } \\
\text { NSCLC }\end{array}$ & None & $\begin{array}{l}\text { XLI84 + } \\
\text { erlotinib }\end{array}$ & 37 & $\begin{array}{l}\text { ORR: } 10.8 \%(95 \% \mathrm{Cl} \\
0.3-21.3 \%) \\
\text { PFS: } 3.6(95 \% \mathrm{Cl} 2.0-5.6) \\
\text { OS: } 13.1 \text { ( } 95 \% \mathrm{Cl} 7.1-\mathrm{NA})\end{array}$ & Completed \\
\hline & NCT0I708954 & 2 & $\begin{array}{l}\text { EGFR wild-type } \\
\text { NSCLC }\end{array}$ & None & $\begin{array}{l}\text { Arm A: } \\
\text { Erlotinib; } \\
\text { Arm B: XLI84; } \\
\text { Arm C: } \\
\text { Erlotinib + } \\
\text { XLI84 }\end{array}$ & $\begin{array}{l}38 \\
38 \\
35\end{array}$ & $\begin{array}{l}\text { Arm A: PFS I.8 }(95 \% \mathrm{Cl} \\
\text { I.7-2.2) } \\
\text { Arm B: PFS } 4.3(95 \% \mathrm{Cl} \\
3.6-7.4) P=0.0003(\mathrm{HR} \\
0.27-0.55) \\
\text { Arm C: PFS } 4.7(95 \% \mathrm{Cl} \\
2.4-7.4) P=0.0003(\mathrm{HR} \\
0.25-0.53)\end{array}$ & $\begin{array}{l}\text { Active, not } \\
\text { recruiting }\end{array}$ \\
\hline & NCT00940225 & 2 & $\begin{array}{l}\text { Selected } \\
\text { tumour } \\
\text { including } \\
\text { NSCLC }\end{array}$ & None & XLI84 & 60 & NSCLC: ORR 10\%; PFS 4.0 & Completed \\
\hline $\begin{array}{l}\text { Foretinib } \\
\text { (GSKI363089) } \\
\text { Multi-TKIs }\end{array}$ & NCTOI068587 & $1 / 2$ & $\begin{array}{l}\text { Pre-treated } \\
\text { advanced } \\
\text { NSCLC } \\
\text { unselected for } \\
\text { EGFR genotype }\end{array}$ & None & $\begin{array}{l}\text { Erlotinib } \pm \\
\text { Foretinib }\end{array}$ & 31 & $\begin{array}{l}\text { ORR: } 17.8 \%(5 / 28) \\
\text { Baseline c-Met expression } \\
\text { associated with response. }\end{array}$ & Completed \\
\hline $\begin{array}{l}\text { Tepotinib } \\
(\text { EMDI2|4063) } \\
\text { selective-TKIs }\end{array}$ & $\begin{array}{l}\text { NCT02864992 } \\
\text { (VISION) }\end{array}$ & 2 & $\begin{array}{l}\text { Advanced } \\
\text { NSCLC }\end{array}$ & $\begin{array}{l}\text { MET Exon } 14 \\
\text { skipping }\end{array}$ & Tepotinib & 85 & $\begin{array}{l}\text { LBx ORR: } 51.4 \% \text { (IR), } 63.9 \% \\
\text { (INV) } \\
\text { TBx ORR: } 41.5 \% \text { (IRC), } 58.5 \% \\
\text { (INV) }\end{array}$ & Recruiting \\
\hline \multirow[t]{2}{*}{$\begin{array}{l}\text { Capmatinib } \\
(\text { INC280, } \\
\text { INCB28060) } \\
\text { selective-TKls }\end{array}$} & NCT0I324479 & 1 & $\begin{array}{l}\text { Advanced solid } \\
\text { tumors } \\
\text { including } \\
\text { NSCLC }\end{array}$ & $\begin{array}{l}\text { CMET }+(H \text {-score } \geq \\
\text { I50 or cMET/ } \\
\text { centromere ratio } \geq \\
2.0 \text { or GCN } \geq 5 \text { or } \\
\text { IHC } 2+\text { or } 3+)\end{array}$ & Capmatinib & 43 & $\begin{array}{l}\text { ORR: } 29 \%(5 / 17) \text { for MET } \\
\text { IHC 3+; } \\
63 \% \text { (5/8) for CMET GCN } \geq 5 \text {; } \\
19 \% \text { (5/26) for MET+. }\end{array}$ & Completed \\
\hline & NCT0I6I0336 & 2 & $\begin{array}{l}\text { Previously } \\
\text { EGFR-TKI } \\
\text { treated EGFR- } \\
\text { mut/C-MET } \\
\text { amplified } \\
\text { NSCLC }\end{array}$ & $\begin{array}{l}\text { MET+ (MET GCN } 4 \\
\text { or } 50 \% \text { of tumor } \\
\text { cells IHC } 3+)\end{array}$ & $\begin{array}{l}\text { INC280 + } \\
\text { gefitinib }\end{array}$ & 83 & $\begin{array}{l}\text { ORR: } \\
18 \%(12 / 65) \text { for total patients } \\
19 \%(10 / 53) \text { for } \mathrm{HC} 2 / 3+/ \\
\mathrm{GCN} \geq 5 \\
30 \%(7 / 23) \text { for } \mathrm{GCN} \geq 6\end{array}$ & $\begin{array}{l}\text { Active, not } \\
\text { recruiting }\end{array}$ \\
\hline
\end{tabular}

(Continued) 
Table I (Continued).

\begin{tabular}{|c|c|c|c|c|c|c|c|c|}
\hline $\begin{array}{l}\text { Agents \& } \\
\text { Mechanism }\end{array}$ & Study & phase & Patients & c-MET Alteration & Treatment & $\begin{array}{l}\text { No. } \\
\text { of } \\
\text { Pts }\end{array}$ & Efficacy Outcome & Status \\
\hline \multirow[t]{5}{*}{$\begin{array}{l}\text { Tivantinib } \\
\text { (ARQ 197) } \\
\text { selective-TKI }\end{array}$} & NCT0I395758 & 2 & $\begin{array}{l}\text { Local advanced } \\
\text { or metastatic } \\
\text { NSCLC }\end{array}$ & None & $\begin{array}{l}\text { ARQ197 + } \\
\text { erlotinib } \\
\text { Pem, Dox, or } \\
\text { Gem }\end{array}$ & $\begin{array}{l}51 \\
45\end{array}$ & $\begin{array}{l}\text { ITT: PFS } 7.3 v s .18 .6, P=0.50 \\
\text { OS } 6.8 v s .8 .5, P=0.44 \\
\text { ORR } 0 \% \text { vs. } 8.9 \%\end{array}$ & Completed \\
\hline & NCT0124419I & 3 & $\begin{array}{l}\text { Local advanced } \\
\text { or metastatic } \\
\text { non-squamous } \\
\text { NSCLC }\end{array}$ & $\begin{array}{l}\text { MET+ }(\mathrm{IHC} \text { score } \geq 2 \\
\text { in } \geq 50 \% \text { tumor } \\
\text { cells; FISH GCN }>4 \text { ) }\end{array}$ & $\begin{array}{l}\text { ARQ197+ } \\
\text { erlotinib } \\
\text { Placebo + } \\
\text { erlotinib }\end{array}$ & $\begin{array}{l}526 \\
522\end{array}$ & $\begin{array}{l}\text { ITT: OS } 8.5 v s .7 .8, P=0.81 \\
\text { PFS 3.6vs. I.9, } P<0.001 \\
\text { ORR I0.3\% vs } 6.5 \% \\
\text { MET +: OS } 9.3 v s .5 .9, P=0.03 \\
\text { PFS } 3.7 v s .1 .9, P=0.01 \\
\text { Longer OS in patients with } \\
\text { tumors with MET GCN }>4 \text { (HR } \\
0.83 \text { ) }\end{array}$ & Completed \\
\hline & NCT0I580735 & 2 & $\begin{array}{l}\text { EGFR mutated, } \\
\text { advanced or } \\
\text { metastatic } \\
\text { NSCLC } \\
\text { progressed on } \\
\text { EGFR-TKI }\end{array}$ & $\begin{array}{l}\text { MET high (IHC } \\
\text { score } \geq 2 \text { in } \geq 50 \% \\
\text { tumour cells; FISH } \\
\geq 4 \text { copies/cells) }\end{array}$ & ARQ197 & 45 & $\begin{array}{l}\text { ITT: ORR } 6.7 \%(95 \% \mathrm{Cl} \text { I. } 4 \% \text { to } \\
\text { 18.3\%) } \\
\text { PFS } 2.7 \text { ( } 95 \% \mathrm{Cl} \text { I.4 to } 4.2) \\
\text { OS } 18.0 \text { ( } 95 \% \mathrm{Cl} \text { I3.4 to } 22.2) \\
\text { c-Met high vs low: PFS } 4.1 \text { vs } \\
\text { I.4; } \\
\text { OS } 20.7 \text { vs } 13.9\end{array}$ & Completed \\
\hline & NCT00777309 & 2 & $\begin{array}{l}\text { Locally } \\
\text { advanced or } \\
\text { metastatic } \\
\text { NSCLC }\end{array}$ & $\begin{array}{l}\text { MET }+ \text { (FISH } \geq 4 \\
\text { copies in } \geq 40 \% \text { of } \\
\text { cells) }\end{array}$ & $\begin{array}{l}\text { Erlotinib } \\
+ \text { ARQ197 } \\
\text { Erlotinib + } \\
\text { placebo }\end{array}$ & $\begin{array}{l}84 \\
83\end{array}$ & $\begin{array}{l}\text { ITT: PFS } 3.8 \text { vs } 2.3, P=0.24 \\
\text { OS } 8.5 \text { vs } 6.9, P=0.47 \\
\text { ORR } 10 \% \text { vs } 7 \%\end{array}$ & Completed \\
\hline & $\begin{array}{l}\text { NCTOI377376 } \\
\text { (ATTENTION) }\end{array}$ & 3 & $\begin{array}{l}\text { LA or } \\
\text { metastatic } \\
\text { NSCLC with } \\
\text { wild type EGFR }\end{array}$ & $\begin{array}{l}\text { MET (IHC score } \geq 2 \\
\text { in } \geq 50 \% \text { tumor } \\
\text { cells; FISH }>4 \\
\text { copies/cells) }\end{array}$ & $\begin{array}{l}\text { ARQ197+ } \\
\text { erlotinib } \\
\text { Placebo + } \\
\text { erlotinib }\end{array}$ & $\begin{array}{l}154 \\
153\end{array}$ & $\begin{array}{l}\text { ITT: OS } 12.7 \text { vs } \mid \mathrm{I} . \mathrm{I}, \mathrm{P}=0.427 \\
\mathrm{PFS} 2.9 \text { vs } 2.0, \mathrm{P}=0.019 \\
\text { Tivantinib was associated with } \\
\text { a weak OS benefit in MET } \\
\mathrm{IHC}+\text { patient (HR } 0.83 \text { ) }\end{array}$ & Terminated* \\
\hline \multirow[t]{2}{*}{$\begin{array}{l}\text { Onartuzumab } \\
\text { (MetMab) }\end{array}$} & $\begin{array}{l}\text { NСT00854308 } \\
\text { (OAM4558g) }\end{array}$ & 2 & $\begin{array}{l}\text { Second- or } \\
\text { third-line } \\
\text { advanced } \\
\text { NSCLC }\end{array}$ & $\begin{array}{l}\text { MET+ (IHC score } 2 \\
+/ 3+)\end{array}$ & $\begin{array}{l}\text { Erlotinib } \\
+ \text { MetMab } \\
\text { Erlotinib } \\
\text { +placebo }\end{array}$ & $\begin{array}{l}69 \\
68\end{array}$ & $\begin{array}{l}\text { All: PFS 2.2vs.2.6, } P=0.69 \\
\text { OS } 8.9 v s .7 .4, P=0.34 \\
\text { ORR 5.8\%vs.4.4\%, } P=0.7 \mid \\
\text { MET+: PFS } 2.9 v s .1 .5, P=0.04 \\
\text { OS } 12.6 v s .3 .8, P=0.002 \\
\text { ORR } 8.6 \% \text { vs.3.2\%, } P=0.37\end{array}$ & Completed \\
\hline & NCT0I456325 & 2 & $\begin{array}{l}\text { Advanced/ } \\
\text { metastatic } \\
\text { NSCLC with } \\
\text { MET diagnosis- } \\
\text { positive }\end{array}$ & $\begin{array}{l}\text { MET+ }(\geq 50 \% \text { of } \\
\text { cells IHC score } 2+ \\
\text { or } 3+; \mathrm{FISH} \geq 5 \\
\text { copies/cell) }\end{array}$ & $\begin{array}{l}\text { Erlotinib + } \\
\text { placebo } \\
\text { Erlotinib+ } \\
\text { MetMab }\end{array}$ & 137 & $\begin{array}{l}\text { OS: FISH+ 2.4vs. I I.I, P=0.35 } \\
\text { IHC+/ FISH-: } 3.7 v s .8 .7, P= \\
0.01 \\
\text { High MET mRNA: } \\
\text { nonsignificant } \\
\text { Low plasma HGF: } 6.9 v s .10 .4 \text {, } \\
\mathrm{p}=0.09\end{array}$ & Completed \\
\hline
\end{tabular}

(Continued) 
Table I (Continued).

\begin{tabular}{|c|c|c|c|c|c|c|c|c|}
\hline $\begin{array}{l}\text { Agents \& } \\
\text { Mechanism }\end{array}$ & Study & phase & Patients & c-MET Alteration & Treatment & $\begin{array}{l}\text { No. } \\
\text { of } \\
\text { Pts }\end{array}$ & Efficacy Outcome & Status \\
\hline & $\begin{array}{l}\text { NCTOI456325 } \\
\text { (MetLung) }\end{array}$ & 3 & $\begin{array}{l}\text { MET-diagnostic } \\
\text { NSCLC }\end{array}$ & $\begin{array}{l}\text { MET+ (IHC score } 2 \\
+/ 3+; \mathrm{FISH} \geq 5 \\
\text { copies/cell) }\end{array}$ & $\begin{array}{l}\text { MetMab+ } \\
\text { erlotinib } \\
\text { Placebo + } \\
\text { erlotinib }\end{array}$ & $\begin{array}{l}250 \\
249\end{array}$ & $\begin{array}{l}\text { ITT: OS 6.8vs.9.1, P=0.067 } \\
\text { PFS } 2.7 v s .2 .6, P=0.92 \\
\text { ORR } 8.4 \% \text { vs.9.6\% } \\
\text { There were no statistically } \\
\text { significant differences in OS, } \\
\text { PFS, or ORR in MET FISH+ } \\
\text { patients. }\end{array}$ & Completed \\
\hline \multirow[t]{2}{*}{$\begin{array}{l}\text { Emibetuzumab } \\
\text { (LY2875358) } \\
\text { MetMab }\end{array}$} & NCTOI900652 & 2 & Stage IV NSCLC & $\begin{array}{l}\text { MET Dx-high }(\geq \\
60 \% \text { of cells } \geq 2+) \\
\text { MET Dx+ }(\text { IHC } \geq \\
10 \% \text { of cells } \geq 2+)\end{array}$ & $\begin{array}{l}\text { Emibetuzumab; } \\
\text { Emibetuzumab } \\
+ \text { erlotinib }\end{array}$ & $\begin{array}{l}28 \\
83\end{array}$ & $\begin{array}{l}\text { MET Dx+ (n=89) } \\
\text { ORR: LY+E: 3.0\% (95\% Cl: } 0.4 \\
+10.5) ; \\
\text { LY: } 4.3 \%(95 \% \text { Cl: } 0.1,21.9) \\
\text { MET Dx-high (n=74) } \\
\text { ORR: LY+E: 3.8\%; LY: 4.8\%. } \\
\text { DCR: LY+E: 50\%; LY: 26\%. } \\
\text { PFS: LY+E: 3.3 (95\% Cl: } \\
\text { I.7-4.2); } \\
\text { LY: I.6 (95\% Cl: I.2-3.I). } \\
\text { OS: LY+E: } 9.2 \text { (95\% Cl: } \\
\text { 6.7-I2.0); } \\
\text { LY: } 8.2 \text { (95\% Cl: 3.7-I2.6). }\end{array}$ & Completed \\
\hline & NCT0I897480 & 2 & $\begin{array}{l}\text { NSCLC with } \\
\text { activating EGFR } \\
\text { mutation }\end{array}$ & $\begin{array}{l}\text { MET IHC } 3+ \\
\text { expression in } \geq 90 \% \\
\text { of tumor cells }\end{array}$ & $\begin{array}{l}\text { Erlotinib; } \\
\text { Erlotinib + } \\
\text { emibetuzumab }\end{array}$ & $\begin{array}{l}70 ; \\
71\end{array}$ & $\begin{array}{l}\text { PFS for ITT: } 9.5 \text { vs } 9.3 \text {, } \\
H R=0.89,90 \% \mathrm{Cl} 0.64-1.23 \text {; } \\
P=0.534 \\
\text { PFS for MET+ }(n=24): \text { PFS } \\
20.7 \text { vs } 5.4, \mathrm{HR}: 0.39 ; 90 \% \mathrm{Cl} \text { : } \\
0.17-0.91\end{array}$ & $\begin{array}{l}\text { Active, not } \\
\text { recruiting }\end{array}$ \\
\hline $\begin{array}{l}\text { Rilotumumab } \\
\text { (AMG 102) } \\
\text { HGBMab }\end{array}$ & NCTOI 233687 & $1 / 2$ & $\begin{array}{l}\text { Previously } \\
\text { treated } \\
\text { advanced } \\
\text { NSCLC }\end{array}$ & none & $\begin{array}{l}\text { Rilotumumab + } \\
\text { Erlotinib }\end{array}$ & 45 & $\begin{array}{l}\text { DCR: } 60 \% \text { (95\% Cl: } 47-71 \%) \\
\text { ORR: } 8.8 \%(95 \% \mathrm{Cl}: \\
\text { 0.4-18.4\%) } \\
\text { PFS: } 2.6 \text { (95\% Cl: I.4-3.3) } \\
\text { OS: } 6.6 \text { (95\% Cl: } 5.6-8.9)\end{array}$ & Completed \\
\hline $\begin{array}{l}\text { Ficlatuzumab } \\
\text { HGBMab }\end{array}$ & NCT0I039948 & 2 & $\begin{array}{l}\text { Lung } \\
\text { adenocarcinoma }\end{array}$ & $\begin{array}{l}\text { MET/HGF } \\
\text { expression (IHC) }\end{array}$ & $\begin{array}{l}\text { Ficlatuzumab + } \\
\text { gefitinib }(G+F) \\
\text { Gefitinib }(G)\end{array}$ & $\begin{array}{l}94 \\
94\end{array}$ & $\begin{array}{l}\text { G+F: ORR } 43 \% \\
\text { PFS } 5.6 \text { for all; } 7.4 \text { for MET } \\
\text { high } \\
\text { G: ORR } 40 \% \\
\text { PFS } 4.7 \text { for all; } 5.5 \text { for MET } \\
\text { high }\end{array}$ & - \\
\hline
\end{tabular}

Note: *Enrollment was stopped when 307 patients were randomized, following the Safety Review Committee's recommendation based on an imbalance in the interstitial lung disease (ILD) incidence between the groups.

Abbreviations: NSCLC, non-small cell lung cancer; ORR, objective response rate; DCR, disease control rate; PFS, progression-free survival; OS, overall survival; MET, mesenchymal-epithelial transition factor; EGFR, epidermal growth factor receptor; ALK, anaplastic lymphoma kinase; IHC, immunohistochemistry; FISH, Fluorescence in situ hybridization; GCN, gene copy number. Pem, Pemetrexed; Dox, docetaxel; Gem, gemcitabine; LBx, liquid biopsy; TBx, tissue biopsy; IRC, independent review committee; INV, Investigator evaluation; LA: local advanced.

\section{Foretinib (XL-880)}

Foretinib is a multi-target kinase inhibitor whose targets include c-Met, ROS-1, RON, VEGFR2, KIT, AXL, TIE2, and PDGFR. ${ }^{108}$ It can also block proliferation and anti- angiogenesis. Recently, a Phase I clinical trial evaluated the efficacy and safety of foretinib in combination with erlotinib in patients with advanced NSCLC who progressed after chemotherapy. ${ }^{108}$ The results showed that 
Table 2 Ongoing Clinical Studies with MET Inhibitors in Advanced NSCLC

\begin{tabular}{|c|c|c|c|c|c|c|}
\hline $\begin{array}{l}\text { Agents \& } \\
\text { mechanism }\end{array}$ & Clinical Trial & Phase & Patients of Lung Cancer & Design & Endpoints & Status \\
\hline \multirow[t]{6}{*}{$\begin{array}{l}\text { Crizotinib } \\
\text { (PF02341066) } \\
\text { Multi-TKIs }\end{array}$} & $\begin{array}{l}\text { NCT00585195 } \\
\text { (PROFILE- } \\
1001 \text { ) }\end{array}$ & 1 & Advanced malignancies including NSCLC & Crizotinib & Safety & $\begin{array}{l}\text { Active, not } \\
\text { recruiting }\end{array}$ \\
\hline & $\begin{array}{l}\text { NCT02465060 } \\
\text { (NCI-MATCH) }\end{array}$ & 2 & $\begin{array}{l}\text { Advanced Refractory Solid Tumors, Lymphomas, or } \\
\text { Multiple Myeloma including lung cancer }\end{array}$ & Crizotinib & Efficacy & Recruiting \\
\hline & $\begin{array}{l}\text { NCT024996I4 } \\
\text { (METROS) }\end{array}$ & 2 & $\begin{array}{l}\text { Pretreated Metastatic Non-small-cell Lung Cancer with } \\
\text { MET Amplification or ROSI Translocation }\end{array}$ & Crizotinib & Efficacy & Recruiting \\
\hline & $\begin{array}{l}\text { NCT02664935 } \\
\text { (Matrix) }\end{array}$ & 2 & NSCLC & Crizotinib & Efficacy & Recruiting \\
\hline & NCT0II 21575 & 1 & NSCLC resistance to erlotinib or gefitinib & $\begin{array}{l}\text { Arml: } \\
\text { Dacomitini } \rightarrow \text { Dacomitinib } \\
\text { +Crizotinib } \\
\text { Arm2: Dacomitinib } \\
\text { +Crizotinib }\end{array}$ & Safety & Completed \\
\hline & NCT0096573I & I & Advanced non-squamous NSCLC & Crizotinib plus erlotinib & Safety & Completed \\
\hline \multirow{5}{*}{$\begin{array}{l}\text { Cabozantinib } \\
(\mathrm{XL}-\mathrm{I84}, \mathrm{BMS}- \\
90735 \mathrm{I}) \\
\text { Multi-TKIs }\end{array}$} & NCT00596648 & $\mathrm{lb} / 2$ & Previously erlotinib treated NSCLC & $\begin{array}{l}\text { Cabotinib with or } \\
\text { without erlotinib }\end{array}$ & $\begin{array}{l}\text { Safety/ } \\
\text { efficacy }\end{array}$ & Completed \\
\hline & NCT039III93 & 2 & NSCLC with MET deregulation & Cabozantinib & Efficacy & Recruiting \\
\hline & NCT0I639508 & 2 & $\begin{array}{l}\text { Advanced NSCLC, ROSI or NTRK Fusions or } \\
\text { Increased MET or AXL Activity }\end{array}$ & Cabozantinib & Efficacy & Recruiting \\
\hline & NCT02132598 & 2 & NSCLC with brain metastases & Cabozantinib & Eficacy & Recruiting \\
\hline & NCT03468985 & 2 & Pre-treated non-squamous NSCLC & $\begin{array}{l}\text { Nivolumab; } \\
\text { Nivolumab + XL-184; } \\
\text { Nivolumab + XL-184+ } \\
\text { ipilimumab }\end{array}$ & Efficacy & $\begin{array}{l}\text { Active, not } \\
\text { recruiting }\end{array}$ \\
\hline $\begin{array}{l}\text { Foretinib } \\
\text { (GSKI363089) } \\
\text { Multi-TKIs }\end{array}$ & NCT02034097 & 2 & Advanced NSCLC & Foretinib \pm erlotinib & Efficacy & Withdrawn \\
\hline \multirow{2}{*}{$\begin{array}{l}\text { Glesatinib } \\
\text { (MGCD265) } \\
\text { Multi-TKIs }\end{array}$} & NCT0295499I & 2 & $\begin{array}{l}\text { Advanced NSCLC, previously treated with platinum } \\
\text { doublet chemotherapy and a checkpoint inhibitor }\end{array}$ & Glesatinib + nivolumab & Efficacy & Recruiting \\
\hline & NCT02544633 & 2 & $\begin{array}{l}\text { Locally advanced or metastatic NSCLC with activating } \\
\text { genetic alterations in MET, including patients with MET } \\
\text { activating mutations in tumor tissue or in blood, and } \\
\text { patients with MET gene amplification in tumor tissue or in } \\
\text { blood. }\end{array}$ & Glesatinib & Efficacy & Completed \\
\hline $\begin{array}{l}\text { Mererstinib } \\
\text { (LY3I64530) } \\
\text { Multi-TKIs }\end{array}$ & NCT02920996 & 2 & $\begin{array}{l}\text { NSCLC Harboring MET Exon } 14 \text { Mutations and Solid } \\
\text { Tumors With NTRK Rearrangements }\end{array}$ & Mererstinib & Efficacy & Recruiting \\
\hline
\end{tabular}

(Continued) 
Table 2 (Continued).

\begin{tabular}{|c|c|c|c|c|c|c|}
\hline $\begin{array}{l}\text { Agents \& } \\
\text { mechanism }\end{array}$ & Clinical Trial & Phase & Patients of Lung Cancer & Design & Endpoints & Status \\
\hline \multirow{3}{*}{$\begin{array}{l}\text { Tepotinib } \\
\text { (EMDI2I4063) } \\
\text { selective-TKIs }\end{array}$} & $\begin{array}{l}\text { NCT0I982955 } \\
\text { (INSIGHT) }\end{array}$ & $1 / 2$ & $\begin{array}{l}\text { Previously EGFR-TKI treated locally advanced or } \\
\text { metastatic EGFR-mutant NSCLC }\end{array}$ & Tepotinib + gefitinib & $\begin{array}{l}\text { Safety/ } \\
\text { efficacy }\end{array}$ & $\begin{array}{l}\text { Active, not } \\
\text { recruiting }\end{array}$ \\
\hline & NCT03940703 & 2 & MET Amplified, Advanced or Metastatic NSCLC & Tepotinib + Osimertinib & $\begin{array}{l}\text { Safety/ } \\
\text { efficacy }\end{array}$ & $\begin{array}{l}\text { Not yet } \\
\text { recruiting }\end{array}$ \\
\hline & $\begin{array}{l}\text { NCT02864992 } \\
(\mathrm{VISION})\end{array}$ & 2 & $\begin{array}{l}\text { Advanced NSCLC with MET Exon } 14 \text { skipping } \\
\text { alterations or MET amplification }\end{array}$ & Tepotinib & Efficacy & Recruiting \\
\hline \multirow{6}{*}{$\begin{array}{l}\text { Savolitinib } \\
\text { (AZD6094, } \\
\text { Volitinib, HMPL- } \\
\text { 504) selective- } \\
\text { TKls }\end{array}$} & NCT02897479 & 2 & NSCLC patients with MET Exon 14 mutation & Savolitinib & Efficacy & Recruiting \\
\hline & $\begin{array}{l}\text { NCT02 } 143466 \\
\text { (TATTON) }\end{array}$ & 1 & $\begin{array}{l}\text { EGFR mutated advanced NSCLC who have progressed } \\
\text { on an EGFR-TKI }\end{array}$ & Savolitinib + osimertinib & Safety & $\begin{array}{l}\text { Active, not } \\
\text { recruiting }\end{array}$ \\
\hline & NCT02374645 & 1 & $\begin{array}{l}\text { EGFR mutated NSCLC who have progressed on EGFR- } \\
\text { TKls }\end{array}$ & Savolitinib + gefitinib & Safety & $\begin{array}{l}\text { Active, not } \\
\text { recruiting }\end{array}$ \\
\hline & NCT03778229 & 2 & $\begin{array}{l}\text { EGFR mutated and MET+ locally advanced or metastatic } \\
\text { NSCLC who have progressed on osimertinib }\end{array}$ & Savolitinib + osimertinib & Efficacy & Recruiting \\
\hline & $\begin{array}{l}\text { NCT03944772 } \\
\text { (ORCHARD) }\end{array}$ & 2 & Advanced NSCLC who have progressed on osimertinib & Savolitinib + osimertinib & Efficacy & Recruiting \\
\hline & $\begin{array}{l}\text { NCT021I7I67 } \\
\text { (SAFIR02_Lung) }\end{array}$ & 2 & $\begin{array}{l}\text { Metastatic NSCLC, MET+ evaluated by throughput } \\
\text { genome analysis }\end{array}$ & Savolitinib & Efficacy & $\begin{array}{l}\text { Active, not } \\
\text { recruiting }\end{array}$ \\
\hline \multirow{10}{*}{$\begin{array}{l}\text { Capmatinib } \\
\text { (INCB28060) } \\
\text { selective-TKIs }\end{array}$} & NCT03693339 & 2 & NSCLC Harboring MET Exon 14 Skipping Mutation & Capmatinib & Efficacy & Recruiting \\
\hline & NCT03647488 & 2 & EGFR-wild and ALK-negative NSCLC & $\begin{array}{l}\text { INC280+spartalizumab; } \\
\text { docetaxel }\end{array}$ & $\begin{array}{l}\text { Safetyl } \\
\text { efficacy }\end{array}$ & Recruiting \\
\hline & NCT03240393 & 2 & Advanced NSCLC & Capmatinib & Efficacy & Withdrawn \\
\hline & NCT02414139 & 2 & EGFR wild-type, advanced NSCLC & Capmatinib & Efficacy & Recruiting \\
\hline & NCT02276027 & 2 & Advanced NSCLC with alteration of the C-MET gene & Capmatinib & Efficacy & $\begin{array}{l}\text { Active, not } \\
\text { recruiting }\end{array}$ \\
\hline & NCT02323126 & 2 & c-Met Positive NSCLC & Capmatinib + nivolumab & Efficacy & Recruiting \\
\hline & NCT02335944 & $1 / 2$ & EGFR-mutant NSCLC & Capmatinib + EGF 816 & $\begin{array}{l}\text { Safety/ } \\
\text { efficacy }\end{array}$ & $\begin{array}{l}\text { Active, not } \\
\text { recruiting }\end{array}$ \\
\hline & NCT01911507 & 1 & C-Met Expressing NSCLC: IHC (2-3+), FISH, RT-PCR & Capmatinib + erlotinib & Safety & $\begin{array}{l}\text { Active, not } \\
\text { recruiting }\end{array}$ \\
\hline & NCT0246866I & 1 & $\begin{array}{l}\text { Advanced/Metastatic NSCLC With EGFR Mutation and } \\
\text { cMET amplification }\end{array}$ & $\begin{array}{l}\text { Capmatinib } \pm \text { erlotinib; } \\
\text { Platinum + pemetrexed }\end{array}$ & Safety & Recruiting \\
\hline & NCT027502I5 & 2 & $\begin{array}{l}\text { NSCLC with MET Exon } 14 \text { Alterations Received Prior } \\
\text { MET Inhibitor }\end{array}$ & Capmatinib & Efficacy & Recruiting \\
\hline \multirow{3}{*}{$\begin{array}{l}\text { Tivantinib (ARQ } \\
\text { 197) selective- } \\
\text { TKls }\end{array}$} & NCT0I069757 & 1 & Advanced/recurrent NSCLC & Tivantinib + erlotinib & Safety & Completed \\
\hline & NCT01251796 & 1 & Advanced/recurrent NSCLC & Tivantinib + erlotinib & & Completed \\
\hline & NCT02049060 & $1 / 2$ & $\begin{array}{l}\text { Advanced/metastatic non-squamous NSCLC } \\
\text { Malignant pleural mesothelioma }\end{array}$ & $\begin{array}{l}\text { Tivantinib + carboplatino } \\
+ \text { pemetrexed }\end{array}$ & Safety & Unknown \\
\hline $\begin{array}{l}\text { SARI } 25844 \\
\text { selective-TKIs }\end{array}$ & NCT02435|2I & 2 & Advanced pre-treated NSCLC with MET amplification & SARI 25844 & Efficacy & Completed \\
\hline
\end{tabular}

(Continued) 
Table 2 (Continued).

\begin{tabular}{|c|c|c|c|c|c|c|}
\hline $\begin{array}{l}\text { Agents \& } \\
\text { mechanism }\end{array}$ & Clinical Trial & Phase & Patients of Lung Cancer & Design & Endpoints & Status \\
\hline \multirow[t]{5}{*}{$\begin{array}{l}\text { Onartuzumab } \\
\text { (MetMAb) }\end{array}$} & NCT0I887886 & 3 & $\begin{array}{l}\text { Stage IIIB/IV NSCLC with EGFR mutation and MET } \\
\text { positive }\end{array}$ & $\begin{array}{l}\text { Erlotinib + onartuzumab } \\
\text { Erlotinib + placebo }\end{array}$ & Efficacy & Completed \\
\hline & NCT0I519804 & 2 & Incurable stage IIIB or IV squamous NSCLC & $\begin{array}{l}\text { Pac/platinum + } \\
\text { Onartuzumab } \\
\text { Pac/platinum + placebo }\end{array}$ & Efficacy & Completed \\
\hline & NCT0I496742 & 2 & Stage IIIB/IV non=squamous NSCLC & $\begin{array}{l}\text { Onartuzumab + Pac/Pem } \\
+ \text { Platinum + Bev } \\
\text { Placebo + Pac/Pem + } \\
\text { Platinum + Bev }\end{array}$ & Efficacy & Completed \\
\hline & NCT0203। 744 & 3 & $\begin{array}{l}\text { Second- or third-line treatment for stage IIIB/IV NSCLC } \\
\text { patients with MET-positive }\end{array}$ & $\begin{array}{l}\text { Erlotinib + placebo } \\
\text { Erlotinib + onartuzumab }\end{array}$ & Efficacy & Completed \\
\hline & $\begin{array}{l}\text { NCT0204460I } \\
\text { (BATTLE-XRT) }\end{array}$ & $1 / 2$ & NSCLC & $\begin{array}{l}\text { Chemoradiation + } \\
\text { onarzumab } \\
\text { Chemoradiation + } \\
\text { onarzumab + erlotinib }\end{array}$ & $\begin{array}{l}\text { Safety/ } \\
\text { efficacy }\end{array}$ & Withdrawn \\
\hline $\begin{array}{l}\text { Telisotuzumab } \\
\text { (ABBV 399) } \\
\text { MetMab }\end{array}$ & $\begin{array}{l}\text { NCT03574753 } \\
\text { (Lung-MAP } \\
\text { SI400K) }\end{array}$ & 2 & $\begin{array}{l}\text { Patients With C-Met Positive Stage IV or Recurrent } \\
\text { Squamous Cell Lung Cancer }\end{array}$ & ABBV-399 & Efficacy & $\begin{array}{l}\text { Active, not } \\
\text { recruiting }\end{array}$ \\
\hline $\begin{array}{l}\text { JNJ-6II86372 } \\
\text { EGFR and MET } \\
\text { antibody }\end{array}$ & NCT02609776 & 1 & Advanced NSCLC & JNJ-61 I86372 & Safety & Recruiting \\
\hline \multirow[t]{2}{*}{$\begin{array}{l}\text { Ficlatuzumab (AV- } \\
\text { 299) HGBMab }\end{array}$} & NCT0I039948 & $\mathrm{lb} / 2$ & Asian NSCLC patients, unselected for EGFR mutation & Ficlatuzumab + gefitinib & $\begin{array}{l}\text { Safetyl } \\
\text { efficacy }\end{array}$ & Completed \\
\hline & NCT023।8368 & 2 & Previously untreated, metastases EGFR-mutant NSCLC & $\begin{array}{l}\text { erlotinib + Ficlatuzumab } \\
\text { Erlotinib + placebo }\end{array}$ & $\begin{array}{l}\text { Safety/ } \\
\text { efficacy }\end{array}$ & Terminated \\
\hline
\end{tabular}

Abbreviations: Bev, Bevacizumab; Pac, Paclitaxel; Pem, Pemetrexed; NSCLC, non-small cell lung cancer; ORR, objective response rate; DCR, disease control rate; PFS, progression-free survival; OS, overall survival; LA, local advanced; MET, mesenchymal-epithelial transition factor; EGFR, epidermal growth factor receptor; ALK, anaplastic lymphoma kinase.

the objective response rate (ORR) of 28 evaluable patients (including wild-type EGFR patients) was $17.8 \%(5 / 28)$. Among the 18 samples, regardless of the EGFR gene status, the baseline MET immunohistochemistry (IHC) expression correlated with the ORR.

MET status evaluated by IHC may be a biomarker for foretinib combined with erlotinib treatment in NSCLC patients who have received chemotherapy. This need further confirmation.

\section{Glesatinib (MGCD265)}

Glesatinib (MGCD265) is an oral type II kinase inhibitor targeting c-Met, VEGFR1, VEGFR2, VEGFR3, TIE2, and RON. ${ }^{4}$ In an ongoing phase I study, ${ }^{109}$ glesatinib showed initial anti-tumor activity in patients with advanced solid tumors with MET positive or AXL rearrangements. All three NSCLC patients with MET alterations (two METex14 mutations, one gene copy number [GCN] increase) showed significant tumor shrinkage at the first assessment.

For NSCLC patients with METex14, glasetinib has shown very promising prospects in phase I clinical trials. This also indicates that METex14 is a potential biomarker. This need to be further confirmed by larger samples.

\section{Selective MET Inhibitors Capmatinib (INCB28060)}

Capmatinib is a highly selective ATP-competitive c-MET inhibitor. It is 10,000 times more selective for c-Met than other kinases, and can inhibits MET activity at picomolar concentrations. ${ }^{110}$ A phase I clinical trial have evaluated the efficacy and safety of capmatinib in NSCLC patients with c-MET positive (H-score $\geq 150$; or c-MET/ 
centromere ratio $\geq 2.0$; or $\mathrm{GCN} \geq 5$; or $\mathrm{IHC} 2+$ or $3+$ ) and EGFR wild type. ${ }^{111}$ A total of 43 patients were included in the NSCLC expansion group. The results showed that in c-MET-positive, cMET IHC $3+$, and cMET GCN $\geq 5$ patients, the ORR were $19 \%(5 / 26), 29 \%(5 / 17)$, and $63 \%$ (5/8), respectively. Another Phase I study evaluated the efficacy and safety of capmatinib as a single-agent therapy in patients with advanced solid tumors (including a group of EGFR wild-type MET+ NSCLC patients; $\mathrm{n}=$ 55). ${ }^{111}$ Preliminary antitumor activity was observed in NSCLC patients with high MET gene copy number ( $\mathrm{GCN} \geq 6$; ORR $=47 \%$ ) or MET overexpression (IHC 3+; $\mathrm{ORR}=24 \%$ ). A phase II study evaluated the efficacy and safety of a combination of capmatinib and gefitinib in patients with EGFR-mutant NSCLC who progressed after gefitinib treatment. ${ }^{112}$ The results showed that in 65 evaluable patients, the ORR was $18 \%$ and the disease control rate (DCR) was $80 \%$. Patients with MET amplification (GCN $\geq 6$ ) obtained an ORR of up to $30 \%$.

A subgroup of a phase II study evaluated the efficacy of capmatinib in METex14+ and EGFR/ALK-negative NSCLC patients (GEOMETRY mono-1), which included a total of six cohorts. Preliminary results showed that for pre-treatment patients with NSCLC with METex4 skipping mutation (cohort 4), capmatinib has a certain ORR and tolerable toxicity, whereas for newly diagnosed patients (cohort 5b) ORR was higher. In 2019, ASCO reported updated data. As of April 15, 2019, a total of 97 patients (cohort 4: $n=69$; cohort $5 \mathrm{~b}$ : $n=28$ ) with NSCLC with METex14 skipping mutations were enrolled. The results showed that for patients in cohort 4 , the ORR was 40.6\% (95\% CI: 28.9-53.1\%), and the median PFS was 5.42 months (95\% CI: 4.17-6.97). For patients in cohort 5b, the ORR was $67.9 \%$ (95\% CI: 47.6-84.1), and the median PFS was 9.69 months (95\% CI: 5.52-13.86). Toxcity was tolerable. In this study, 13 patients had baseline brain metastases, and the intracranial ORR was 54\% (7/13), of these, 4 patients achieved complete response (CR); the intracranial DCR was 92\% (12/13). The response time of internal lesion was consistent with the response time of the extracranial lesion.

These results indicate that MET amplification and METex14 may be potential biomarkers for the treatment of capmatinib in patients with NSCLC. Capmatinib appears to be more effective in newly diagnosis NSCLC patients with METex14.

\section{Tivantinib (ARQ 197)}

Tivantinib is a non-ATP competitive c-MET inhibitor. A randomized, double-blind, open-label phase II trial evaluated the efficacy and safety of erlotinib in combination with tivantinib versus erlotinib in combination with placebo in patients with advanced NSCLC. A total of 167 patients were enrolled: the proportion of patients harboring EGFR mutations in the combination and standard treatment groups was $10 \%$ and $18 \%$, respectively, and the proportion of patients with MET GCN $\geq 4$ was $26 \%$ and $26.5 \%$, respectively. Compared to standard treatment group, the combination treatment group had a valueadvantaged ORR (10\% vs 7\%), median PFS (3.8 vs 2.3 months; HR $=0.81 ; \mathrm{p}=0.24)$, and $\operatorname{mOS}(8.5$ vs 6.9 months; hazard ratio $[\mathrm{HR}]=0.87 ; \mathrm{p}=0.47$ ), but there was no statistical difference. An exploratory analysis of non-squamous NSCLC showed a benefit trend for PFS $(\mathrm{HR}=0.71)$ and overall survival $(\mathrm{HR}=0.72)$ in the combination group compared with the standard treatment group. Subgroup analysis showed that patients with EGFR wild-type $(\mathrm{HR}=0.70)$, KRAS mutation $(\mathrm{HR}=0.76)$, and MET FISH-positive $(>5, \mathrm{HR}=0.45)$ had an advantage with PFS. In the two groups, both were safely tolerated. ${ }^{113}$

The MARQUEE phase III study evaluated the efficacy and safety of erlotinib in combination with tivantinib vs erlotinib in combination with placebo in patients with pre-treated NSCLC. ${ }^{114}$ A total of 1048 patients were enrolled. Interim analysis showed that combination therapy improved median PFS (3.6 vs 1.9 months; HR-0.74; 95\% CI: 0.62-0.89; $\mathrm{p}<0.001$ ) but did not improve mOS (8.5 vs 7.8 months; $\mathrm{HR}=0.98$; 95\% CI: $0.84-1.15$ ) in intention-to-treat (ITT) patients. Exploratory subgroup analysis showed that the combination therapy prolonged $\mathrm{mOS}(\mathrm{HR}=0.70 ; 95 \% \mathrm{CI}$ : 0.49-1.01) in patients with high MET expression (MET GCN $\geq 4$ ). This result was confirmed by a phase III study in Asian patients with previously treated stage IIIB/IV non-squamous NSCLC harboring wild-type EGFR (ATTENTION study). ${ }^{115}$ This study began enrollment with a target of 460 patients. Due to an imbalance in the incidence of interstitial lung disease (ILD) between the 2 groups, 307 patients with NSCLC were finally randomized to receive either erlotinib in combination with tivantinib or erlotinib in combination with placebo. ILD occurred in 6 patients $(0$ deaths $)$ and 14 patients ( 3 deaths) in the placebo and tivantinib group, respectively. In ITT population, compared to placebo group, the tivantinib group had significantly prolonged PFS (2.9 months vs 2.0 months; $\mathrm{HR}=0.719 ; \mathrm{p}=0.019$ ), and had 
a value-advantaged overall survival (12.7 months and 11.1 months; $\mathrm{HR}=0.891 ; \mathrm{p}=0.427$ ).

These results indicate that for patients with NSCLC, MET amplification evaluated by FISH appears to be a potential biomarker for the treatment of tivantinib in combination of erlotinib.

\section{Savolitinib (Volitinib, AZD6094, HMPL-504)}

Savolitinib is a selective small molecule MET inhibitor that blocks c-Met activity in an ATP-dependent way. It has high selectivity for MET. ${ }^{116}$ A phase II clinical trial evaluated the efficacy and safety of savolitinib in combination with osimertinib in patients with NSCLC with positive T790M mutations resistance to osimertinib. ${ }^{117}$ Preliminary results showed that of the seven patients who received combination therapy, two obtained PR, with tolerable safety.

Osimertinib combined with savolitinib may be a therapy strategy for patients with NSCLC who are resistant to osimertinib. These may show better clinical efficacy in patients with MET alterations after osimertinib resistance. However, further confirmation is needed.

\section{Tepotinib (EMDI2 | 4063)}

Tepotinib is an oral, ATP-competitive, highly selective c-MET TKI. Its selectivity to c-Met is 1000 times that of other kinases. ${ }^{118}$ A phase II study evaluated the efficacy and safety of epotinib in patients with NSCLC harboring METEx14 mutations and EGFR/ALK wild-type. The primary end point was the ORR assessed by the independent review committee (IRC) while the secondary endpoint was ORR and safety evaluated by the investigator (INV). As at the time of the data analysis, 85 patients were enrolled (55 and 52 patients in the liquid biopsy [LBx] and biopsy group $[\mathrm{TBX}]$, respectively). Thirty-five patients in the LBx group were evaluated for efficacy, the ORR, evaluated by IRC, was $51.4 \%$ and $63.9 \%$ evaluated by INV. Forty-one patients in the TBx group underwent efficacy evaluation, the ORR was $41.5 \%$ evaluated by IRC and $58.5 \%$ evaluated by INV. The toxicity was tolerable.

These results indicate that for patients with NSCLC, METex14 maybe a biomarker for tepotinib treatment.

\section{Anti-MET Antibodies Onartuzumab (MetMAb)}

Onartuzumab is a fully humanized, c-MET monoclonal antibody that blocks the alpha chain binding of the HGF to the c-MET ligand binding domain. ${ }^{119}$ In a phase II study, ${ }^{120}$ pretreated NSCLC patients were randomized into two groups, one receiving onartuzumab in combination with erlotinib and the other receiving placebo plus erlotinib. A total of 137 patients were enrolled in this study, of which 26 patients (23\%) harbored KRAS mutations while 13 patients (12\%) harbored EGFR mutations. The results showed that compared with placebo group, the onartuzumab group showed no prolonged $\mathrm{PFS}(\mathrm{HR}=1.09 ; \mathrm{P}=0.69)$ and overall survival $(\mathrm{HR}=0.80 ; \mathrm{P}=0.34)$ in the ITT population. In MET-positive patients $(n=66)$, PFS $(H R=0.53 ; p=0.04)$ and overall survi$\operatorname{val}(\mathrm{HR}=0.37 ; \mathrm{p}=0.002)$ were significantly prolonged in the onartuzumab group. However, in MET-negative patients $(\mathrm{n}=62)$, PFS $(\mathrm{HR}=1.82, \mathrm{p}=0.05)$ and overall survival $(\mathrm{HR}=1.78, \mathrm{p}=0.16)$ were poor in the onartuzumab group. MET-positive patients in the placebo group had a worse PFS $(\mathrm{HR}=1.71 ; \mathrm{p}=0.06)$ and overall survival $(\mathrm{HR}=2.61$; $\mathrm{p}=0.004$ ) than MET-negative patients. Thus, in METpositive people, onartuzumab plus erlotinib can improve PFS and overall survival, while in MET-negative patients, the combination therapy is even worse. Therefore, a larger randomized trial included 499 pre-treated patients with METpositive NSCLC. ${ }^{121}$ They were randomized (1:1) to receive erlotinib in combination with placebo or erlotinib in combination with onartuzumab. The results showed that patients in the onartuzumab group did not have improved overall survival (6.8 vs 9.1 months; $H R=1.27 ; p=0.067)$, PFS ( 2.7 vs 2.6 months; HR=0.99; $\mathrm{p}=0.92)$, and overall $\mathrm{RR}(8.4 \%$ vs $9.6 \%$; $\mathrm{p}=0.63)$. The trial has been terminated.

Larger clinical trials have not confirmed better efficacy of onartuzumab combined with erlotinib in patients with MET-positive NSCLC. The screening of biomarkers may need to be explored.

\section{Emibetuzumab (LY2875358)}

Emibetuzumab is a c-MET monoclonal antibody that blocks the binding of HGF and c-MET, leading to internalization and degradation of c-MET, and prevention of signal transmission. A phase II clinical trial (NCT01900652) evaluated the efficacy and safety of emibetuzumab with or without erlotinib in patients with METpositive stage IV NSCLC. ${ }^{122}$ A total of 111 patients were enrolled in this study, of which 28 patients received emibetuzumab monotherapy, and 83 patients received emibetuzumab plus erlotinib. The results showed that in patients with MET Dx $+(\geq 10 \%$ of cells $\geq 2+$ by IHC, $n=89)$ the ORR of monotherapy group was $4.3 \%$ (95\% CI: $0.1-$ $21.9 \%$ ), and the ORR of the combination treatment group was 3.0\% (95\% CI: $0.4-10.5 \%)$. In MET Dx-high population ( $\geq 60 \%$ of cells $\geq 2+; n=74$ ), the combination 
treatment group had longer PFS (3.3 vs 1.6 months) and overall survival (9.2 vs 8.2 months), and lower ORR $(3.8 \%$ vs $4.8 \%)$ than the monotherapy group. Another phase II clinical trial (NCT01897480) evaluated the efficacy and safety of erlotinib with or without emibetuzumab in patients with advanced NSCLC harboring EGFR mutations. $^{123}$ A total of 141 patients were enrolled, of which 71 patients were treated with combination therapy and 70 patients received erlotinib monotherapy. The results showed that in the ITT population, the combination treatment group had advantaged value PFS (9.5 vs 9.3 months; HR=0.89; 90\% CI 0.64-1.23; $\mathrm{P}=0.534$ ), with no statistical difference. Exploratory analysis showed that in patients with MET high expression ( $\geq 90 \%$ of cells MET IHC $3+; \mathrm{n}=24)$, the combination treatment significantly prolonged PFS (20.7 vs 5.4 months; HR=0.39; 90\% CI: 0.17-0.91) compared to emibetuzumab monotherapy. In the rest of the population, PFS was not statistically different $(\mathrm{HR}=1.1 ; 90 \% \mathrm{CI}: 0.7-1.7)$.

These results indicate that MET IHC status may be a potential biomarker for the treatment of emibetuzumab in patients with NSCLC.

\section{Telisotuzumab (ABBV 399)}

Telisotuzumab is a novel anti-c-MET antibody drug conjugate that can be coupled to monomethyl staphylococin E (MMAE), which mediates killing requiring a threshold level of c-MET expression. ${ }^{124}$ In a phase I study, 16 patients with MET-positive (IHC H score $\geq 150$ ) NSCLC were treated with ABBV-399. ${ }^{125}$ The results showed that 3 patients $(18.8 \%$; 95\% CI: 4.1\%-45.7\%) obtained PR. The median response duration time was 4.8 months, and the median PFS was 5.7 months (95\% CI, 1.2-15.4 months). The toxicity was tolerable.

Telisotumumab has certain effect on NSCLC patients with MET IHC positive, further confirmation is needed.

\section{JNJ-6I I 86372}

JNJ-372 can block ligand binding by binding to EGFR and MET, promote receptor degradation, and trigger antibodydependent cytotoxicity in the EGFR mutant (EGFRm) NSCLC model. ${ }^{126}$ A phase I study evaluated the efficacy and safety of JIN-61186372 in patients with advanced NSCLC harboring EGFR mutations. In 2019, ASCO congress reported the interim results. Up to the cut-off data analysis, a total of 116 patients were enrolled in the study, of which $97 \%$ harbored EGFR mutations. In 88 evaluable patients, $28 \%(25 / 88)$ achieved PR. In 47 patients who were resistant to third generation EGFR-TKI, 21.3\% (10/47) achieved PR (6 confirmed), 4 of whom were C797S, 1 was MET amplification, and 5 had no identifiable EGFR/MET gene alterations. In 20 patients with EGFR 20ins, 30\% (6/20) achived PR (3 confirmed). The toxicity was tolerable. Patients with resistance to third generation EGFR-TKI, including C797S, MET amplification, and exon 20ins, all had initial responses, and research on expanded doses are ongoing.

JNJ-61186372 has clinical activity in NSCLC patients who harbor EGFR mutation or who are resistant to the third-generation EGFR-TKI, regardless of whether or not the cause of resistance is due to MET amplification.

\section{Anti-HGF Antibodies}

Rilotumumab (AMG-I02)

Rilotumumab is a fully humanized $\mathrm{IgG} 2$ monoclonal antibody that binds to the HGF $\beta$ chain and inhibits HGF binding to c-MET. $^{127,128}$ A Phase 1/2 study evaluated the efficacy and safety of rilotumumab in combination with erlotinib in patients with NSCLC regardless of the EGFR status. ${ }^{129}$ In all evaluable patients $(\mathrm{n}=45)$, the ORR was $8.8 \%$ (90\% CI: $0.4-18.4 \%)$, the DCR was $60 \%$ (95\% CI: 47-71\%), the median PFS was 2.6 months (90\% CI: $1.4-3.3$ months) and the mOS was 6.6 months (90\% CI: 5.6-8.9 months). For patients with wild-type EGFR $(n=33)$, the DCR was $60.6 \% \quad(90 \%$ CI: 46.3-73.3\%), the median PFS was 2.6 months (90\% CI: 1.4-2.7 months), and the mOS was 7.0 months (90\% CI: 5.6-13.4 months).

The results suggested that blocking HGF combined with EGFR-TKI may increase the efficacy of NSCLC patients with EGFR wild-type.

\section{Ficlatuzumab (AV-299)}

Ficlatuzumab is a high-affinity humanized IgG1 monoclonal antibody directed against HGF that inhibits HGFinduced c-MET signaling pathway by blocking $\mathrm{HGF} /$ cMET binding. ${ }^{130}$ A randomized phase II clinical trial evaluated the efficacy of gefitinib with or without ficlatuzumab in patients with NSCLC. ${ }^{131}$ In the EGFR mutations and low c-MET expression subgroup, patients receiving ficlatuzumab plus gefitinib had an improvement in ORR (41\% vs 22\%) and median PFS (11 vs 5.5 months). However, in the ITT population, the combination therapy did not significantly improve ORR ( $40 \%$ vs $38 \%, \mathrm{P}=0.77$ ), PFS (5.6 vs 4.7 months, $\mathrm{HR}=0.94, \mathrm{P}=0.67$ ) and overall survival (24.7 vs 21.8 months, $\mathrm{HR}=0.98, \mathrm{P}=0.91$ ) compared with the gefitinib monotherapy group. 
Ficlatuzumab plus gefitinib can improve the clinical efficacy in NSCLC patients with EGFR mutations and low c-MET expression, indicating that MET expression maybe a biomarker for the ficlatuzumab treatment.

\section{Immunotherapy}

For patients with NSCLC with MET alterations, immunotherapy can be administered after exhaustion of the targeted therapy and chemotherapy. When to start immunotherapy for these patients; however, remains to be explored. A study retrospectively analyzed the clinical activity of immunotherapy in patients with NSCLC with oncogenic driver alterations, including 36 patients with MET alterations (MET amplification $\mathrm{n}=13$, METex14 skipping mutation $n=23) .{ }^{132}$ They were all received immunotherapy in different treatment lines. In MET alteration subgroup analysis, ORR, median PFS and OS were 16\%, 3.4 months and 18.4 months, respectively. Another study retrospectively included 147 patients to evaluate the PD-L1 expression, tumor mutational burden (TMB), and clinical activity of immunotherapy in patients with METex14 lung cancers. The PD-L1 positive (PD-L1 $\geq 1 \%$ ) rate was $63 \%(57 / 111)$, while TMB was lower in tumors with METex14 alterations compared with unselected NSCLCs. In 24 patients who received immunotherapy, the ORR was $17 \%$, and the median OS was 18.2 months. The median PFS was 1.9 months for the 21 patients assessable for this end point. ${ }^{133}$ Therefore, compared with the targeted agents and chemotherapy, immunotherapy had a poor clinical activity in MET alteration patients with NSCLC. Single-agent immunotherapy should not be considered before receiving targeted therapies and chemotherapy. The role of combination treatment (ie immunotherapy and chemotherapy, immunotherapy and targeted therapy, immunotherapy and antiangiogenic agents, or combination immunotherapy) in MET alteration patients with NSCLC need further investigation.

\section{Future Challenges}

In recent phase III clinical trials, the efficacy of MET inhibitors to treat NSCLC was disappointed. ${ }^{114,115,134}$ Whether the target agents were inhibiting the c-MET receptor itself or inhibiting its ligand HGF, they were not effective as monotherapy for patients with unselected NSCLC. From the current research results, the subgroups of patients with NSCLC harboring MET gene alterations, especially MET amplification or METex14 skipping mutation, respond to MET inhibitors. Therefore, it is important to find appropriate biomarkers for selecting patients. In order to improve the applicability of biomarkers, it is important to set two thresholds that distinguish between MET positive and negative or between high and low HGF, and to choose the appropriate method to achieve this. To solve this problem, there is need for large data analysis for the detection methods and treatment effects, to establish standards that meet the MET activation status, and determine reliable thresholds to achieve effective patient stratification and clinical decision making.

In fact, MET inhibitors cannot block the growth of tumors without MET gene alterations, which does not mean that MET inhibitors are not effective in this case. When MET inhibitors are combined with kinase inhibitors in the upstream or downstream signaling pathways, they can inhibit parallel kinase signaling from other receptors, which may improve the clinical outcomes. However, the toxicity of targeted combination therapies could be considerable. In addition, since MET is related to the properties of activated dendritic cell tolerogenic, MET inhibitors may act synergistically with immunological checkpoint inhibitors such as PD-1 antibodies to restore the immune stimulating microenvironment, and release the tumoricidal activity of cytotoxic $\mathrm{T}$ cells. Other combination therapies include HGF/c-Met inhibitors plus inhibitors that inhibit the vitality or function of stromal cells such as tumorassociated fibroblasts, endothelial cells, or macrophages.

\section{Conclusion}

The MET/HGF axis is a promising therapeutic target in advanced NSCLC. A variety of MET inhibitors have been developed, some of which have entered phase III clinical trials. However, the results of phase III clinical trials to date have been disappointing. Various mechanisms of MET activation in lung cancer, including MET/HGF overexpression, MET gene alterations (such as mutations, amplification, translocation, or transcriptional disorders), and impaired MET degradation provide a range of potential biomarkers. The challenge now is to use a range of agents currently under development to determine which biomarkers are most likely to select the appropriate patient for MET-targeted therapy.

\section{Disclosure}

The authors report no conflicts of interest in this work.

\section{References}

1. Siegel RL, Miller KD, Jemal A. Cancer statistics, 2017. Ca-Cancer J Clin. 2017;67:7-30. doi:10.3322/caac.21387 
2. Burdett S, Stephens R, Stewart L, et al. Chemotherapy in addition to supportive care improves survival in advanced non-small-cell lung cancer: a systematic review and meta-analysis of individual patient data from 16 randomized controlled trials - NSCLC meta-analyses collaborative group. J Clin Oncol. 2008;26:4617-4625.

3. Tsao AS, Scagliotti GV, Bunn PA, et al. Scientific advances in lung cancer 2015. J Thoracic Oncol. 2016;11:613-638. doi:10.1016/j. jtho.2016.03.012

4. Birchmeier C, Birchmeier W, Gherardi E, et al. Met, metastasis, motility and more. Nature Rev Mol Cell Biol. 2003;4:915-925. doi: $10.1038 / \mathrm{nrm} 1261$

5. Blumenschein GR, Mills GB, Gonzalez-angulo AM. Targeting the hepatocyte growth factor-cMET axis in cancer therapy. $J$ Clin Oncol. 2012;30:3287-3296. doi:10.1200/JCO.2011.40.3774

6. Rosario M, Birchmeier W. How to make tubes: signaling by the Met receptor tyrosine kinase. Trends Cell Biol. 2003;13:328-335. doi:10.1016/S0962-8924(03)00104-1

7. Gentile A, Trusolino L, Comoglio PM. The Met tyrosine kinase receptor in development and cancer. Cancer Metast Rev. 2008;27:85-94. doi:10.1007/s10555-007-9107-6

8. Zhang YW, Su YL, Volpert OV, et al. Hepatocyte growth factor/ scatter factor mediates angiogenesis through positive VEGF and negative thrombospondin 1 regulation. Proc Natl Acad Sci USA. 2003;100:12718-12723. doi:10.1073/pnas.2135113100

9. Olivero M, Rizzo M, Madeddu R, et al. Overexpression and activation of hepatocyte growth factor scatter factor in human non-smallcell lung carcinomas. Brit J Cancer. 1996;74:1862-1868. doi:10.10 38/bjc. 1996.646

10. Ma PC, Tretiakova MS, MacKinnon AC, et al. Expression and mutational analysis of MET in human solid cancers. Gene Chromosome Canc. 2008;47:1025-1037. doi:10.1002/gcc.20604

11. Beau-faller M, Ruppert AM, Voegeli AC, et al. MET gene copy number in non-small cell lung cancer: molecular analysis in a targeted tyrosine kinase inhibitor naive cohort. $J$ Thoracic Oncol. 2008;3:331-339. doi:10.1097/JTO.0b013e318168d9d4

12. Masuya D, Huang C, Liu D, et al. The tumour-stromal interaction between intratumoral c-Met and stromal hepatocyte growth factor associated with tumour growth and prognosis in non-small-cell lung cancer patients. Brit J Cancer. 2004;90:1555-1562. doi:10.1038/sj.bjc.6601718

13. Cappuzzo F, Marchetti A, Skokan M, et al. Increased MET gene copy number negatively affects survival of surgically resected non-small-cell lung cancer patients. $J$ Clin Oncol. 2009;27:1667-1674. doi:10.1200/JCO.2008.19.1635

14. Dimou A, Non L, Chae YK, et al. MET gene copy number predicts worse overall survival in patients with Non-Small Cell Lung Cancer (NSCLC); A systematic review and meta-analysis. PLoS One. 2014;9.

15. Guo BP, Cen H, Tan XH, et al. Prognostic value of MET gene copy number and protein expression in patients with surgically resected non-small cell lung cancer: a meta-analysis of published literatures. PLoS One. 2014;9.

16. Christensen JG, Zou HY, Arango ME, et al. Cytoreductive antitumor activity of PF-2341066, a novel inhibitor of anaplastic lymphoma kinase and c-Met, in experimental models of anaplastic large-cell lymphoma. Mol Cancer Ther. 2007;6:3314-3322. doi:10.1158/1535-7163.MCT-07-0365

17. Sadiq AA, Salgia R. MET as a possible target for non-small-cell lung cancer. J Clin Oncol. 2013;31:1089-1096. doi:10.1200/ JCO.2012.43.9422

18. Peters S, Adjei AA. MET: a promising anticancer therapeutic target. Nat Rev Clin Oncol. 2012;9:314-326. doi:10.1038/ nrclinonc.2012.71

19. Basile JR, Afkhami T, Gutkind JS. Semaphorin 4D/Plexin-B1 induces endothelial cell migration through the activation of PYK2, Src, and the phosphatidylinositol 3-kinase-Akt pathway. Mol Cell Biol. 2005;25:6889-6898. doi:10.1128/MCB.25.16.68896898.2005
20. Maroun CR, Naujokas MA, Holgado-madruga M, et al. The tyrosine phosphatase SHP-2 is required for sustained activation of extracellular signal-regulated kinase and epithelial morphogenesis downstream from the Met receptor tyrosine kinase. Mol Cell Biol. 2000;20:8513-8525. doi:10.1128/MCB.20.22.8513-8525.2000

21. Lee YY, Kim HP, Kang MJ, et al. Phosphoproteomic analysis identifies activated MET-axis PI3K/AKT and MAPK/ERK in lapatinib-resistant cancer cell line. Exp Mol Med. 2013;45:e64. doi: $10.1038 / \mathrm{emm} .2013 .115$

22. Scagliotti GV, Novello S, von Pawel J. The emerging role of MET/ HGF inhibitors in oncology. Cancer Treat Rev. 2013;39:793-801. doi:10.1016/j.ctrv.2013.02.001

23. Organ SL, Tsao MS. An overview of the c-MET signaling pathway. Ther Adv Med Oncol. 2011;3:S7-S19. doi:10.1177/1758834011422556

24. Finocchiaro G, Toschi L, Gianoncelli L, et al. Prognostic and predictive value of MET deregulation in non-small cell lung cancer. Ann Transl Med. 2015;3:83.

25. Birchmeier C, Birchmeier W, Gherardi E, et al. Met, metastasis, motility and more. Nat Rev Mol Cell Bio. 2003;4:915-925. doi: $10.1038 / \mathrm{nrm} 1261$

26. Trusolino L, Bertotti A, Comoglio PM. MET signalling: principles and functions in development, organ regeneration and cancer. Nat Rev Mol Cell Bio. 2010;11:834-848. doi:10.1038/nrm3012

27. Appleman LJ. MET signaling pathway: a rational target for cancer therapy. J Clin Oncol. 2011;29:4837-4838. doi:10.1200/JCO.2011.37. 7929

28. Gherardi E, Sandin S, Petoukhov MV, et al. Structural basis of hepatocyte growth factor/scatter factor and MET signalling. Proc Natl Acad Sci USA. 2006;103:4046-4051. doi:10.1073/pnas.050 9040103

29. Zhang YW, Woude GFV. HGF/SF-Met signaling in the control of branching morphogenesis and invasion. $J$ Cell Biochem. 2003;88:408-417. doi:10.1002/jcb. 10358

30. Christensen JG, Burrows J, Salgia R. c-Met as a target for human cancer and characterization of inhibitors for therapeutic intervention. Cancer Lett. 2005;225:1-26. doi:10.1016/j.canlet.2004.09.044

31. Bean J, Brennan C, Shih JY, et al. MET amplification occurs with or without T790M mutations in EGFR mutant lung tumors with acquired resistance to gefitinib or erlotinib. Proc Natl Acad Sci U S A. 2007;104:20932-20937. doi:10.1073/pnas.0710370104

32. Engelman JA, Zejnullahu K, Mitsudomi T, et al. MET amplification leads to gefitinib resistance in lung cancer by activating ERBB3 signaling. Science. 2007;316:1039-1043. doi:10.1126/science.1141478

33. Kong-beltran M, Seshagiri S, Zha JP, et al. Somatic mutations lead to an oncogenic deletion of Met in lung cancer. Cancer Res. 2006;66:283-289. doi:10.1158/0008-5472.CAN-05-2749

34. Weidner KM, Sachs M, Riethmacher D, et al. Mutation of juxtamembrane tyrosine residue-1001 suppresses loss-of-function mutations of the met receptor in epithelial-cells. Proc Natl Acad Sci USA. 1995;92:2597-2601. doi:10.1073/pnas.92.7.2597

35. Peschard P, Fournier TM, Lamorte L, et al. Mutation of the c-Cbl TKB domain binding site on the Met receptor tyrosine kinase converts it into a transforming protein. Mol Cell. 2001;8:995-1004. doi:10.1016/ S1097-2765(01)00378-1

36. Ma PC, Jagadeeswaran R, Jagadeesh S, et al. Functional expression and mutations of c-met and its therapeutic inhibition with SU11274 and small interfering RNA in non-small cell lung cancer. Cancer Res. 2005;65:1479-1488. doi:10.1158/0008-5472. CAN-04-2650

37. Collisson EA, Campbell JD, Brooks AN, et al. Comprehensive molecular profiling of lung adenocarcinoma. Nature. 2014; 511:543-550.

38. Paik PK, Drilon A, Fan PD, et al. Response to MET inhibitors in patients with stage IV lung adenocarcinomas harboring MET mutations causing exon 14 skipping. Cancer Discov. 2015;5:842-849. doi:10.1158/2159-8290.CD-14-1467 
39. Awad MM, Oxnard GR, Jackman DM, et al. MET exon 14 mutations in non-small-cell lung cancer are associated with advanced age and stage-dependent MET genomic amplification and c-met overexpression. J Clin Oncol. 2016;34:721. doi:10.1200/JCO.2015.63.4600

40. Heist RS, Shim HS, Gingipally S, et al. MET exon 14 skipping in non-small cell lung cancer. Oncologist. 2016;21:481-486. doi:10.1634/ theoncologist.2015-0510

41. Schrock AB, Frampton GM, Suh J, et al. Characterization of 298 patients with lung cancer harboring MET exon 14 skipping alterations. J Thorac Oncol. 2016;11:1493-1502. doi:10.1016/j. jtho.2016.06.004

42. Frampton GM, Ali SM, Rosenzweig M, et al. Activation of MET via diverse exon 14 splicing alterations occurs in multiple tumor types and confers clinical sensitivity to MET inhibitors. Cancer Discov. 2015;5:850-859. doi:10.1158/2159-8290.CD-15-0285

43. Kalluri R. The biology and function of fibroblasts in cancer. Nature Rev Cancer. 2016;16:582-598. doi:10.1038/nrc.2016.73

44. Onozato R, Kosaka T, Kuwano H, et al. Activation of MET by gene amplification or by splice mutations deleting the juxtamembrane domain in primary resected lung cancers. $J$ Thoracic Oncol. 2009;4:5-11. doi:10.1097/JTO.0b013e3181913e0e

45. Seo JS, Ju YS, Lee WC, et al. The transcriptional landscape and mutational profile of lung adenocarcinoma. Genome Res. 2012;22:2109-2119. doi:10.1101/gr.145144.112

46. Saffroy R, Fallet V, Girard N, et al. MET exon 14 mutations as targets in routine molecular analysis of primary sarcomatoid carcinoma of the lung. Oncotarget. 2017;8:42428-42437. doi:10.18632/ oncotarget. 16403

47. Liu XW, Jia YX, Stoopler MB, et al. Next-generation sequencing of pulmonary sarcomatoid carcinoma reveals high frequency of actionable MET gene mutations. J Clin Oncol. 2016;34:794 doi:10.1200/JCO.2015.62.0674

48. Vuong HG, Ho ATN, Altibi AMA, et al. Clinicopathological implications of MET exon 14 mutations in non-small cell lung cancer A systematic review and meta-analysis. Lung Cancer. 2018;123: 76-82. doi:10.1016/j.lungcan.2018.07.006

49. Tong JH, Yeung SF, Chan AWH, et al. MET amplification and exon 14 splice site mutation define unique molecular subgroups of non-small cell lung carcinoma with poor prognosis. Clin Cancer Res. 2016;22:3048-3056. doi:10.1158/1078-0432.CCR-15-2061

50. Drilon AE, Camidge DR, Ou SHI, et al. Efficacy and safety of crizotinib in patients (pts) with advanced MET exon 14-altered non-small cell lung cancer (NSCLC). J Clin Oncol. 2016;34. doi:10.1200/JCO.2016.34.15_suppl.108

51. Lee C, Usenko D, Frampton GM, et al. MET 14 deletion in sarcomatoid non-small-cell lung cancer detected by next-generation sequencing and successfully treated with a MET inhibitor. J Thoracic Oncol. 2015;10:E113-E114. doi:10.1097/ JTO.0000000000000645

52. Waqar SN, Morgensztern D, Sehn J. MET mutation associated with responsiveness to crizotinib. J Thoracic Oncol. 2015;10:E29-E31. doi:10.1097/JTO.0000000000000478

53. Mendenhall MA, Goldman JW. MET-mutated NSCLC with major response to crizotinib. J Thoracic Oncol. 2015;10:E33-E34. doi:10.1097/JTO.0000000000000491

54. Jenkins RW, Oxnard GR, Elkin S, et al. Response to crizotinib in a patient with lung adenocarcinoma harboring a MET splice site mutation. Clin Lung Cancer. 2015;16:E101-E104. doi:10.1016/j. cllc.2015.01.009

55. Drilon A, Cappuzzo F, Ou S-HI, et al. Targeting MET in lung cancer: will expectations finally be MET? J Thoracic Oncol. 2017;12:15-26. doi:10.1016/j.jtho.2016.10.014

56. Zheng Z, Liebers M, Zhelyazkova B, et al. Anchored multiplex PCR for targeted next-generation sequencing. Nat Med. 2014;20:1479-1484. doi:10.1038/nm.3729
57. Rodgers K, Network CGAR. Comprehensive molecular profiling of lung adenocarcinoma (vol 511, pg 543, 2014). Nature. 2014;514.

58. Kawakami H, Okamoto I, Okamoto W, et al. Targeting MET amplification as a new oncogenic driver. Cancers. 2014;6:1540-1552. doi:10.3390/cancers6031540

59. Noonan SA, Berry L, Lu X, et al. Identifying the appropriate FISH criteria for defining MET copy number-driven lung adenocarcinoma through oncogene overlap analysis. J Thoracic Oncol. 2016;11:1293-1304. doi:10.1016/j.jtho.2016.04.033

60. Chen HJ, Mok TS, Chen ZH, et al. Clinicopathologic and molecular features of epidermal growth factor receptor T790M mutation and c-MET amplification in tyrosine kinase inhibitor-resistant Chinese non-small cell lung cancer. Pathol Oncol Res. 2009;15:651-658. doi:10.1007/s12253-009-9167-8

61. Go H, Jeon YK, Park HJ, et al. High MET gene copy number leads to shorter survival in patients with non-small cell lung cancer. J Thoracic Oncol. 2010;5:305-313. doi:10.1097/JTO.0b013e3181ce3d1d

62. Caparica R, Yen CT, Coudry R, et al. Responses to crizotinib can occur in high-level MET-amplified non-small cell lung cancer independent of MET exon 14 alterations. J Thorac Oncol. 2017;12:141-144. doi:10.1016/j.jtho.2016.09.116

63. Ou SHI, Kwak EL, Siwak-tapp C, et al. Activity of crizotinib (PF02341066), a dual mesenchymal-epithelial transition (MET) and anaplastic lymphoma kinase (ALK) inhibitor, in a non-small cell lung cancer patient with de novo MET amplification. J Thoracic Oncol. 2011;6:942-946. doi:10.1097/JTO.0b013e31821528d3

64. Camidge DR, Ou SHI, Shapiro G, et al. Efficacy and safety of crizotinib in patients with advanced c-MET-amplified non-small cell lung cancer (NSCLC). J Clin Oncol. 2014;32:8001. doi:10.1200/jco.2014.32.15_suppl.8001

65. Zhang Y, Wang WY, Wang Y, et al. Response to crizotinib observed in lung adenocarcinoma with MET Copy number gain but without a high-level MET/CEP7 ratio, MET overexpression, or exon 14 splicing mutations. J Thoracic Oncol. 2016;11:E59-E62. doi:10.1016/ j.jtho.2015.12.102

66. Gherardi E, Birchmeier W, Birchmeier C, et al. Targeting MET in cancer: rationale and progress. Nature Rev Cancer. 2012;12:89-103. doi:10.1038/nrc3205

67. Abella JV, Peschard P, Naujokas MA, et al. Met/hepatocyte growth factor receptor ubiquitination suppresses transformation and is required for Hrs phosphorylation. Mol Cell Biol. 2005;25:9632-9645. doi:10.1128/MCB.25.21.9632-9645.2005

68. Tan YHC, Mirzapoiazova T, Won BM, et al. Differential responsiveness of MET inhibition in non-small-cell lung cancer with altered CBL. Sci Rep. 2017;7:1-3.

69. Yeh I, Botton T, Talevich E, et al. Activating MET kinase rearrangements in melanoma and Spitz tumours. Nat Commun. 2015;6. doi: $10.1038 /$ ncomms 8174

70. Davies KD, Ng TL, Estrada-bernal A, et al. Dramatic response to crizotinib in a patient with lung cancer positive for an HLA-DRB1MET gene fusion. JCO Precis Oncol. 2017;2017.

71. Stransky N, Cerami E, Schalm S, et al. The landscape of kinase fusions in cancer. Nat Commun. 2014;5. doi:10.1038/ncomms5846

72. Kentsis A, Reed C, Rice KL, et al. Autocrine activation of the MET receptor tyrosine kinase in acute myeloid leukemia. Nat Med. 2012;18:1118. doi:10.1038/nm.2819

73. Danilkovitch-miagkova A, Zbar B. Dysregulation of Met receptor tyrosine kinase activity in invasive tumors. $J$ Clin Invest. 2002;109:863-867. doi:10.1172/JCI0215418

74. Park S, Choi YL, Sung CO, et al. High MET copy number and MET overexpression: poor outcome in non-small cell lung cancer patients. Histol Histopathol. 2012;27:197-207. doi:10.14670/HH-27.197

75. Tsao M-S, Yang Y, Marcus A, et al. Hepatocyte growth factor is predominantly expressed by the carcinoma cells in non-small-cell lung cancer. Hum Pathol. 2001;32:57-65. doi:10.1053/hupa. 2001.21133 
76. Tsuta K, Kozu Y, Mimae T, et al. c-MET/phospho-MET protein expression and MET gene copy number in non-small cell lung carcinomas. J Thorac Oncol. 2012;7:331-339. doi:10.1097/JTO. 0b013e318241655f

77. Tretiakova M, Salama AKS, Karrison T, et al. MET and phosphorylated MET as potential biomarkers in lung cancer. $J$ Environ Pathol Tox. 2011;30:341-354. doi:10.1615/JEnvironPatholToxicolOncol.v30.i4.70

78. Ye S, Li JK, Hao K, et al. The efficacy and risk profile of c-Met inhibitors in non-small cell lung cancer: a meta-analysis. Sci Rep. 2016;6. doi:10.1038/srep35770

79. Lee GD, Lee SE, Oh DY, et al. MET exon 14 skipping mutations in lung adenocarcinoma: clinicopathologic implications and prognostic values. $J$ Thoracic Oncol. 2017;12:1233-1246. doi:10.1016/j. jtho.2017.04.031

80. Kwon D, Koh J, Kim S, et al. MET exon 14 skipping mutation in triple-negative pulmonary adenocarcinomas and pleomorphic carcinomas: an analysis of intratumoral MET status heterogeneity and clinicopathological characteristics. Lung Cancer. 2017;106:131-137. doi:10.1016/j.lungcan.2017.02.008

81. Casadevall D, Gimeno J, Clave S, et al. MET expression and copy number heterogeneity in nonsquamous non-small cell lung cancer (nsNSCLC). Oncotarget. 2015;6:16215-16226. doi:10.18632/ oncotarget.v6i18

82. Scagliotti GV, Novello S, Schiller JH, et al. Rationale and design of MARQUEE: a phase III, randomized, double-blind study of tivantinib plus erlotinib versus placebo plus erlotinib in previously treated patients with locally advanced or metastatic, nonsquamous, non-small-cell lung cancer. Clin Lung Cancer. 2012;13:391-395. doi:10.1016/j.cllc.2012.01.003

83. Spigel DR, Edelman MJ, Mok T, et al. Treatment rationale study design for the metlung trial: a randomized, double-blind phase III study of onartuzumab (MetMAb) in combination with erlotinib versus erlotinib alone in patients who have received standard chemotherapy for stage IIIB or IV met-positive non-small-cell lung cancer. Clin Lung Cancer. 2012;13:500-504. doi:10.1016/j. cllc.2012.05.009

84. Turke AB, Zejnullahu K, Wu YL, et al. Preexistence and clonal selection of MET amplification in EGFR mutant NSCLC. Cancer Cell. 2010;17:77-88. doi:10.1016/j.ccr.2009.11.022

85. Papadimitrakopoulou VA, Wu YL, Han JY, et al. Analysis of resistance mechanisms to osimertinib in patients with EGFR T790M advanced NSCLC from the AURA3 study. Ann Oncol. 2018;29:741. doi:10.1093/annonc/mdy424.064

86. Ramalingam SS, Cheng Y, Zhou C, et al. Mechanisms of acquired resistance to first-line osimertinib: preliminary data from the phase III FLAURA study. Ann Oncol. 2018;29:viii740. doi:10.1093/ annonc/mdy 424.063

87. Yano $\mathrm{S}$, Wang $\mathrm{W}, \mathrm{Li}$ Q, et al. Hepatocyte growth factor induces gefitinib resistance of lung adenocarcinoma with epidermal growth factor receptor-activating mutations. Cancer Res. 2008;68:9479-9487. doi:10.1158/0008-5472.CAN-08-1643

88. Jiang W, Hiscox S, Matsumoto K, et al. Hepatocyte growth factor scatter factor, its molecular, cellular and clinical implications in cancer. Crit Rev Oncol Hemat. 1999;29:209-248. doi:10.1016/ S1040-8428(98)00019-5

89. Kosaka T, Yamaki E, Mogi A, et al. Mechanisms of resistance to EGFR TKIs and development of a new generation of drugs in non-small-cell lung cancer. J Biomed Biotechnol. 2011;2011:1-7. doi: $10.1155 / 2011 / 165214$

90. Bardelli A, Corso S, Bertotti A, et al. Amplification of the MET receptor drives resistance to anti-EGFR therapies in colorectal cancer. Cancer Discov. 2013;3:658-673. doi:10.1158/2159-8290.CD-12-0558

91. Pietrantonio F, Oddo D, Gloghini A, et al. MET-driven resistance to dual EGFR and BRAF blockade may be overcome by switching from EGFR to MET inhibition in BRAF-mutated colorectal cancer. Cancer Discov. 2016;6:963-971. doi:10.1158/2159-8290.CD-16-0297
92. Puri N, Salgia R. Synergism of EGFR and c-Met pathways, cross-talk and inhibition, in non-small cell lung cancer. J Carcinog. 2008;7:9. doi:10.4103/1477-3163.44372

93. Benvenuti S, Gentile A, Lazzari L, et al. An 'in-cell trial' to assess the efficacy of a monovalent anti-MET antibody as monotherapy and in association with standard cytotoxics. Mol Oncol. 2014;8:378-388. doi:10.1016/j.molonc.2013.12.006

94. Awad MM, Leonardi GC, Kravets S, et al. Impact of MET inhibitors on survival among patients with non-small cell lung cancer harboring MET exon 14 mutations: a retrospective analysis. Lung Cancer. 2019;133:96-102. doi:10.1016/j.lungcan. 2019.05.011

95. Schrock AB, Frampton GM, Suh J, et al. Characterization of 298 patients with lung cancer harboring MET exon 14 skipping alterations. J Thoracic Oncol. 2016;11:1493-1502. doi:10.1016/j. jtho.2016.06.004

96. Liu SY, Gou LY, Li AN, et al. The unique characteristics of MET exon 14 mutation in chinese patients with NSCLC. $J$ Thoracic Oncol. 2016;11:1503-1510. doi:10.1016/j.jtho.2016.05.016

97. Zou HY, Li QH, Lee JH, et al. An orally available small-molecule inhibitor of c-met, PF-2341066, exhibits cytoreductive antitumor efficacy through antiproliferative and antiangiogenic mechanisms. Cancer Res. 2007;67:4408-4417. doi:10.1158/0008-5472.CAN-064443

98. Cui JJ, Tran-dube M, Shen H, et al. Structure based drug design of crizotinib (PF-02341066), a potent and selective dual inhibitor of mesenchymal-epithelial transition factor (c-MET) kinase and anaplastic lymphoma kinase (ALK). J Med Chem. 2011;54:6342-6363. doi:10.1021/jm2007613

99. Malik SM, Maher VE, Bijwaard KE, et al. US food and drug administration approval: crizotinib for treatment of advanced or metastatic non-small cell lung cancer that is anaplastic lymphoma kinase positive. Clin Cancer Res. 2014;20:2029-2034. doi:10.1158/ 1078-0432.CCR-13-3077

100. Kazandjian D, Blumenthal GM, Luo L, et al. Benefit-risk summary of crizotinib for the treatment of patients with ROS1 alteration-positive, metastatic non-small cell lung cancer. Oncologist. 2016;21:974-980. doi:10.1634/theoncologist.2016-0101

101. Awad MM, Leonardi GC, Kravets S, et al. Impact of MET inhibitors on survival among patients (pts) with MET exon 14 mutant (METdel14) non-small cell lung cancer (NSCLC). J Clin Oncol. 2017;35:8511. doi:10.1200/JCO.2017.35.15_suppl.8511

102. Ou SHI, Govindan R, Eaton KD, et al. Phase I results from a study of crizotinib in combination with erlotinib in patients with advanced nonsquamous non-small cell lung cancer. $J$ Thoracic Oncol. 2017;12:145-151. doi:10.1016/j.jtho.2016.09.131

103. Janne PA, Shaw AT, Camidge DR, et al. Combined pan-HER and ALK/ROS1/MET inhibition with dacomitinib and crizotinib in advanced non-small cell lung cancer: results of a phase I study. $J$ Thoracic Oncol. 2016;11:737-747. doi:10.1016/j.jtho.2016. 01.022

104. Taniguchi H, Yamada T, Takeuchi S, et al. Impact of MET inhibition on small-cell lung cancer cells showing aberrant activation of the hepatocyte growth factor/MET pathway. Cancer Sci. 2017;108:1378-1385. doi:10.1111/cas.2017.108.issue-7

105. Schoffski P, Gordon M, Smith DC, et al. Phase II randomised discontinuation trial of cabozantinib in patients with advanced solid tumours. Eur J Cancer. 2017;86:296-304. doi:10.1016/j. ejca.2017.09.011

106. Reckamp KL, Mack PC, Ruel N, et al. Biomarker analysis of a phase II trial of cabozantinib and erlotinib in patients (pts) with EGFR-mutant NSCLC with epidermal growth factor receptor (EGFR) tyrosine kinase inhibitor (TKI) resistance: a California Cancer Consortium phase II trial (NCI 9303). J Clin Oncol. 2015;33:8087. doi:10.1200/jco.2015.33.15_suppl.8087 
107. Neal JW, Dahlberg SE, Wakelee HA, et al. Erlotinib, cabozantinib, or erlotinib plus cabozantinib as second-line or third-line treatment of patients with EGFR wild-type advanced non-small-cell lung cancer (ECOG-ACRIN 1512): a randomised, controlled, open-label, multicentre, Phase 2 trial. Lancet Oncol. 2016;17:1661-1671. doi:10.1016/ S1470-2045(16)30561-7

108. Leighl NB, Tsao MS, Liu G, et al. A phase I study of foretinib plus erlotinib in patients with previously treated advanced non-small cell lung cancer: Canadian cancer trials group IND.196. Oncotarget. 2017;8:69651-69662. doi:10.18632/oncotarget.18753

109. Kollmannsberger CK, Sharma S, Shapiro G, et al. Phase I study of receptor tyrosine kinase (RTK) inhibitor, MGCD265, in patients (pts) with advanced solid tumors. J Clin Oncol. 2015;33:2589. doi:10.1200/jco.2015.33.15 supp1.2589

110. Liu XD, Wang Q, Yang GJ, et al. A novel kinase inhibitor, INCB28060, blocks c-MET-dependent signaling, neoplastic activities, and cross-talk with EGFR and HER-3. Clin Cancer Res. 2011;17:7127-7138. doi:10.1158/1078-0432.CCR-11-1157

111. Schuler MH, Berardi R, Lim WT, et al. Phase (Ph) I study of the safety and efficacy of the cMET inhibitor capmatinib (INC280) in patients (pts) with advanced cMET plus non-small cell lung cancer (NSCLC). J Clin Oncol. 2016;34:9067. doi:10.1200/JCO.2016.34.15_suppl.9067

112. Wu YL, Kim DW, Felip E, et al. Phase (Ph) II safety and efficacy results of a single-arm ph ib/II study of capmatinib (INC280) + gefitinib in patients (pts) with EGFR-mutated (mut) cMET-positive (cMET plus) non-small cell lung cancer (NSCLC). J Clin Oncol. 2016;34:9020. doi:10.1200/JCO.2016.34.15_suppl.9020

113. Sequist LV, von Pawel J, Garmey EG, et al. Randomized phase II study of erlotinib plus tivantinib versus erlotinib plus placebo in previously treated non-small-cell lung cancer. J Clin Oncol. 2011;29:3307-3315. doi:10.1200/JCO.2010.34.0570

114. Scagliotti G, von Pawel J, Novello S, et al. Phase III multinational, randomized, double-blind, placebo-controlled study of tivantinib (ARQ 197) plus erlotinib versus erlotinib alone in previously treated patients with locally advanced or metastatic nonsquamous non-small-cell lung cancer. $J$ Clin Oncol. 2015;33:2667. doi:10.1200/JCO.2014.60.7317

115. Yoshioka H, Azuma K, Yamamoto N, et al. A randomized, double-blind, placebo-controlled, phase III trial of erlotinib with or without a c-Met inhibitor tivantinib (ARQ 197) in Asian patients with previously treated stage IIIB/IV nonsquamous nonsmall-cell lung cancer harboring wild-type epidermal growth factor receptor (ATTENTION study). Ann Oncol. 2015;26:2066-2072. doi:10. 1093/annonc/mdv288

116. Jia H, Dai GX, Weng JY, et al. Discovery of (S)-1-(1-(Imidazo [1,2-a]pyridin-6-yl)ethyl)-6-(1-methyl-1H-pyrazol-4-yl)-1H-[1,2,3] triazolo[4,5-b]pyrazine (Volitinib) as a highly potent and selective mesenchymal-epithelial transition factor (c-Met) inhibitor in clinical development for treatment of cancer. $J$ Med Chem. 2014;57:7577-7589. doi:10.1021/jm500510f

117. Oxnard GR, Ramalingam SS, Ahn MJ, et al. Preliminary results of TATTON, a multi-arm phase Ib trial of AZD9291 combined with MEDI4736, AZD6094 or selumetinib in EGFR-mutant lung cancer. J Clin Oncol. 2015;33:2509. doi:10.1200/jco.2015.33.15_suppl.2509

118. Bladt F, Faden B, Friese-hamim M, et al. EMD 1214063 and EMD 1204831 constitute a new class of potent and highly selective c-met inhibitors. Clin Cancer Res. 2013;19:2941-2951. doi:10.1158/ 1078-0432.CCR-12-3247

119. Merchant M, Ma XL, Maun HR, et al. Monovalent antibody design and mechanism of action of onartuzumab, a MET antagonist with anti-tumor activity as a therapeutic agent. Proc Natl Acad Sci USA. 2013;110:E2987-E2996. doi:10.1073/pnas.1302725110

120. Spigel DR, Ervin TJ, Ramlau RA, et al. Randomized phase II trial of onartuzumab in combination with erlotinib in patients with advanced non-small-cell lung cancer. J Clin Oncol. 2013;31:4105. doi:10.1200/JCO.2012.47.4189
121. Spigel DR, Edelman MJ, O'Byrne K, et al. Results from the phase III randomized trial of onartuzumab plus erlotinib versus erlotinib in previously treated stage IIIB or IV non-small-cell lung cancer: mETLung. J Clin Oncol. 2017;35:412. doi:10.1200/JCO.2016. 69.2160

122. Camidge DR, Moran T, Demedts I, et al. A randomized, open-label, phase 2 study of emibetuzumab plus erlotinib (LY plus E) and emibetuzumab monotherapy (LY) in patients with acquired resistance to erlotinib and MET diagnostic positive (MET Dx plus) metastatic NSCLC. J Clin Oncol. 2016;34:9070. doi:10.1200/ JCO.2016.34.15_suppl.9070

123. Scagliotti GV, Moro-sibilot D, Kollmeier J, et al. A randomized, controlled, open label phase II study of erlotinib (E) with or without the MET antibody emibetuzumab (Emi) as first-line treatment for EGFRmt non-small cell lung cancer (NSCLC) patients who have disease control after an 8-week lead-in treatment with erlotinib. J Clin Oncol. 2017;35.

124. Wang JY, Anderson MG, Oleksijew A, et al. ABBV-399, a c-met antibody-drug conjugate that targets both MET-amplified and c-met-overexpressing tumors, irrespective of MET pathway dependence. Clin Cancer Res. 2017;23:992-1000. doi:10.1158/ 1078-0432.CCR-16-1568

125. Strickler JH, Weekes CD, Nemunaitis J. First-in-human phase I, dose-escalation and -expansion study of telisotuzumab vedotin, an antibody-drug conjugate targeting c-met, in patients with advanced solid tumors (vol 36, pg 3298, 2018). J Clin Oncol. 2019;37:261.

126. Emdal KB, Dittmann A, Reddy RJ, et al. Characterization of in vivo resistance to osimertinib and JNJ-61186372, an EGFR/ met bispecific antibody, reveals unique and consensus mechanisms of resistance. Mol Cancer Ther. 2017;16:2572-2585. doi:10.1158/ 1535-7163.MCT-17-0413

127. Burgess TL, Sun J, Meyer S, et al. Biochemical characterization of AMG 102: a neutralizing, fully human monoclonal antibody to human and nonhuman primate hepatocyte growth factor. Mol Cancer Ther. 2010;9:400-409. doi:10.1158/1535-7163.MCT-09-0824

128. Giordano $\mathrm{S}$. Rilotumumab, a mAb against human hepatocyte growth factor for the treatment of cancer. Curr Opin Mol Ther. 2009; $11: 448-455$.

129. Tarhini AA, Rafique I, Floros T, et al. Phase $1 / 2$ study of rilotumumab (AMG 102), a hepatocyte growth factor inhibitor, and erlotinib in patients with advanced non-small cell lung cancer. Cancer. 2017;123:2936-2944. doi:10.1002/cncr.v123.15

130. D'arcangelo M, Cappuzzo F. Focus on the potential role of ficlatuzumab in the treatment of non-small cell lung cancer. Biologics. 2013;7:61-68.

131. Mok TSK, Geater SL, Su WC, et al. A randomized phase 2 study comparing the combination of ficlatuzumab and gefitinib with gefitinib alone in asian patients with advanced stage pulmonary adenocarcinoma. J Thoracic Oncol. 2016;11:1736-1744. doi:10. 1016/j.jtho.2016.05.038

132. Mazieres J, Drilon A, Lusque A, et al. Immune checkpoint inhibitors for patients with advanced lung cancer and oncogenic driver alterations: results from the IMMUNOTARGET registry. Ann Oncol. 2019;30:1321-1328. doi:10.1093/annonc/mdz167

133. Sabari JK, Leonardi GC, Shu CA, et al. PD-L1 expression, tumor mutational burden, and response to immunotherapy in patients with MET exon 14 altered lung cancers. Ann Oncol. 2018;29:2085-2091. doi:10.1093/annonc/mdy334

134. Spigel DR, Edelman MJ, O’byrne K, et al. Onartuzumab plus erlotinib versus erlotinib in previously treated stage IIIb or IV NSCLC: results from the pivotal phase III randomized, multicenter, placebo-controlled METLung (OAM4971g) global trial. $J$ Clin Oncol. 2014;32:8000. doi:10.1200/jco.2014.32.15_sup $\mathrm{pl} .8000$ 


\section{Publish your work in this journal}

OncoTargets and Therapy is an international, peer-reviewed, open access journal focusing on the pathological basis of all cancers, potential targets for therapy and treatment protocols employed to improve the management of cancer patients. The journal also focuses on the impact of management programs and new therapeutic agents and protocols on patient perspectives such as quality of life, adherence and satisfaction. The manuscript management system is completely online and includes a very quick and fair peer-review system, which is all easy to use. Visit http://www.dovepress.com/ testimonials.php to read real quotes from published authors.

Submit your manuscript here: https://www.dovepress.com/oncotargets-and-therapy-journal 